\title{
Ice nucleation activity of silicates and aluminosilicates in pure water and aqueous solutions - Part 2: Quartz and amorphous silica
}

\author{
Anand Kumar, Claudia Marcolli, and Thomas Peter \\ Institute for Atmospheric and Climate Sciences, ETH Zurich, Zurich, 8092, Switzerland
}

Correspondence: Anand Kumar (anand.kumar@env.ethz.ch)

Received: 26 September 2018 - Discussion started: 13 November 2018

Revised: 25 March 2019 - Accepted: 11 April 2019 - Published: 8 May 2019

\begin{abstract}
Divergent ice nucleation (IN) efficiencies of quartz, an important component of atmospheric mineral dust, have been reported in previous studies. We show here that quartz particles obtain their IN activity from milling and that quartz aged in water loses most of its IN efficiency relative to freshly milled quartz. Since most studies so far reported IN activities of commercial quartz dusts that were milled already by the manufacturer, IN active samples prevailed. Also, the quartz surface - much in contrast to that of feldspars - is not prone to ammonia-induced IN enhancement. In detail we investigate the influence of solutes on the IN efficiency of various silica $\left(\mathrm{SiO}_{2}\right)$ particles (crystalline and amorphous) with special focus on quartz. We performed immersion freezing experiments and relate the observed variability in IN activity to the influence of milling, the aging time and to the exposure conditions since milling. Immersion freezing with silica particles suspended in pure water or aqueous solutions of $\mathrm{NH}_{3},\left(\mathrm{NH}_{4}\right)_{2} \mathrm{SO}_{4}, \mathrm{NH}_{4} \mathrm{HSO}_{4}, \mathrm{Na}_{2} \mathrm{SO}_{4}$ and $\mathrm{NaOH}$, with solute concentrations corresponding to water activities $a_{\mathrm{w}}=0.9-1.0$, were investigated in emulsified droplets by means of differential scanning calorimetry (DSC) and analyzed in terms of the onset temperature of the heterogeneous freezing signal $T_{\text {het }}$ and the heterogeneously frozen water volume fraction $F_{\text {het }}$. Quartz particles, which originate from milling coarse samples, show a strong heterogeneous freezing peak in pure water with $T_{\text {het }}$ equal to $247-$ $251 \mathrm{~K}$. This IN activity disappears almost completely after aging for 7 months in pure water in a glass vial. During this time quartz slowly grew by incorporating silicic acid leached from the glass vial. Conversely, the synthesized amorphous silica samples show no discernable heterogeneous freezing signal unless they were milled. This implies that defects provide IN activity to silica surfaces, whereas the IN activity
\end{abstract}

of a natural quartz surface is negligible, when it grew under near-equilibrium conditions. For suspensions containing milled quartz and the solutes $\left(\mathrm{NH}_{4}\right)_{2} \mathrm{SO}_{4}, \mathrm{NH}_{4} \mathrm{HSO}_{4}$ or $\mathrm{Na}_{2} \mathrm{SO}_{4}, T_{\text {het }}$ approximately follows $T_{\text {het }}^{\Delta a_{\mathrm{w}}^{\text {het }}}\left(a_{\mathrm{w}}\right)$, the heterogeneous freezing onset temperatures that obey $\Delta a_{\mathrm{w}}^{\text {het }}$ criterion, i.e., $T_{\text {het }}^{\Delta a_{\mathrm{w}}^{\text {het }}}\left(a_{\mathrm{w}}\right)=T_{\text {melt }}\left(a_{\mathrm{w}}+\Delta a_{\mathrm{w}}^{\text {het }}\right)$ with $\Delta a_{\mathrm{w}}^{\text {het }}$ being a constant offset with respect to the ice melting point curve, similar to homogeneous IN. This water-activity-based description is expected to hold when the mineral surface is not altered by the presence of the solutes. On the other hand, we observe a slight enhancement in $F_{\text {het }}$ in the presence of these solutes, implying that the compliance with the $\Delta a_{\mathrm{w}}^{\text {het }}$ criterion does not necessarily imply constant $F_{\text {het }}$. In contrast to the sulfates, dilute solutions of $\mathrm{NH}_{3}$ or $\mathrm{NaOH}$ (molality $\geq 5 \times 10^{-4} \mathrm{~mol} \mathrm{~kg}^{-1}$ ) reveal $T_{\text {het }}$ by $3-8 \mathrm{~K}$ lower than $T_{\text {het }}^{\Delta a_{\mathrm{w}}^{\text {het }}}\left(a_{\mathrm{w}}\right)$, indicating a significant impact on the mineral surface. The lowering of $T_{\text {het }}$ of quartz suspended in dilute $\mathrm{NH}_{3}$ solutions is opposite to the distinct increase in $T_{\text {het }}$ that we found in emulsion freezing experiments with aluminosilicates, namely feldspars, kaolinite, gibbsite and micas. We ascribe this decrease in IN activity to the increased dissolution of quartz under alkaline conditions. The defects that constitute the active sites appear to be more susceptible to dissolution and therefore disappear first on a dissolving surface.

\section{Introduction}

The influence of cirrus and mixed-phase clouds on Earth's radiative budget is well recognized, yet not fully understood (Baker, 1997; DeMott et al., 2010; Storelvmo et al., 2011). 
Ice formation in clouds may be initiated via homogeneous ice nucleation (IN) below $237 \mathrm{~K}$, whereas it requires an ice nucleating particle (INP) to occur heterogeneously at higher temperatures between 237 and $273 \mathrm{~K}$ (Pruppacher and Klett, 1994; Vali et al., 2015). Mineral dusts are a well-established class of aerosol particles, consisting of various minerals, such as feldspars, clay minerals, micas, calcite and quartz, which exhibit widely varying IN abilities (Murray et al., 2011; Atkinson et al., 2013; Kaufmann et al., 2016). The atmospheric relevance of these different minerals as INPs depends on both their abundance in airborne dusts and their IN activity, which in turn may depend on their production process and atmospheric aging.

For a long time, clay minerals have been considered the dominating IN active species amongst mineral dust particles. This is because of their well-documented IN ability together with their high abundance in the fine particle fraction, which facilitates long-range and high-altitude transport (Usher et al., 2003; Matsuki et al., 2005; Murray et al., 2012; Pinti et al., 2012). However, they are IN active only at temperatures too low to explain many observed instances of cloud glaciation (Atkinson et al., 2013). More recently, feldspars, and more specifically potassium-containing feldspars (Kfeldspars) have been suggested as the determinant species for the IN activity of airborne desert dusts (Atkinson et al., 2013). Yet, follow-up studies have shown that not all $\mathrm{K}$ feldspars exhibit the same high IN activity (Harrison et al., 2016; Kaufmann et al., 2016; Peckhaus et al., 2016), and that microcline, the K-feldspar with the highest freezing temperatures, constitutes only a minor fraction of collected desert dusts (Boose et al., 2016b; Kaufmann et al., 2016).

Quartz, the dominant dust component collected near source regions, is a crystalline mineral composed of silicon and oxygen atoms in a continuous framework of $\mathrm{SiO}_{4}$ tetrahedra, with each oxygen atom being shared by two tetrahedra. Therefore, quartz has an overall chemical formula of silicon dioxide $\left(\mathrm{SiO}_{2}\right)$, also called silica (Götze and Möckel, 2014). Quartz is a potentially relevant mineral dust for heterogeneous IN in the atmosphere (Field et al., 2006; Murray et al., 2012; Boose et al., 2016b). Moreover, it is found in high proportions in atmospherically transported Saharan dust samples (Avila et al., 1997; Caquineau et al., 1998; 2002; Alastuey et al., 2005; Kandler et al., 2009). Boose et al. (2016b) found a correlation of IN activity with the quartz concentration in dust samples, which they collected either after being airborne and transported or directly at the surface from deserts worldwide, suggesting that quartz particles have the potential to be relevant INPs for cloud glaciation in the atmosphere. Indeed, quartz particles showed IN activity in laboratory studies, albeit with very different IN efficiencies (Pruppacher and Sänger, 1955; Isono and Ikebe, 1960; Zimmermann et al., 2008; Atkinson et al., 2013; Zolles et al., 2015; Kaufmann et al., 2016). Some early studies including Pruppacher and Sänger (1955) and Isono and Ikebe (1960) found quartz to be IN active in their experiments. For su- permicrcon quartz particles immersed in pure water, Zimmermann et al. (2008) reported an activated fraction of $1 \%$ at $261 \mathrm{~K}\left(\mathrm{RH}_{\mathrm{W}}>100 \%\right)$. In droplet freezing experiments, Atkinson et al. (2013) and Zolles et al. (2015) found $50 \%$ of droplets frozen as high as $249 \mathrm{~K}$ and as low as $235 \mathrm{~K}$, depending on sample origin and pretreatment (e.g., milling). These examples show that although quartz has a simple chemical composition, the quartz surface seems to show large variations with respect to its surface properties resulting in highly variable IN activities.

Mineral surfaces may undergo changes due to interaction with atmospheric chemical species while being transported over long distances (Prospero, 1999; Schepanski et al., 2009; Uno et al., 2009). These changes can potentially alter their IN ability (Salam et al., 2007, 2008; Kulkarni et al., 2012; Augustin-Bauditz et al., 2014). Several studies reported an indifferent behavior of mineral surfaces to dissolved species so that immersion freezing in solutions can be simply described as a freezing point depression due to the solute $(\mathrm{Zu}-$ beri et al., 2002; Zobrist et al., 2008; Rigg et al., 2013), with a constant offset in water activity ( $\Delta a_{\mathrm{w}}=$ const.), similar to the water-activity-based description of homogeneous IN by Koop et al. (2000). This description was further elaborated as the activity-based immersion freezing model (ABIFM) by Knopf and Alpert (2013). In contrast, other studies have shown that IN efficiencies in immersion mode deteriorated due to irreversible surface destruction (e.g., in the presence of acids) (Niedermeier et al., 2011; Augustin-Bauditz et al., 2014; Wex et al., 2014; Burkert-Kohn et al., 2017) as well as in deposition mode (Sullivan et al., 2010b; Reitz et al., 2011). However, Sullivan et al. (2010a) showed that size-selected Arizona Test Dust (ATD) exposed to nitric acid resulted in hampering of IN efficiency deposition mode but had no impact on freezing above water saturation. On the other hand, Kanji et al. (2019) reported no effect of secondary organic aerosol (SOA) coating on the IN efficiency of Saharan and Asian dust samples in immersion freezing mode.

This is the second part of three companion papers on IN activity of silicates and aluminosilicates. In Part 1 (Kumar et al., 2018a) we have shown that immersion freezing onset temperatures of microcline in aqueous solutions strongly deviate from a constant $\Delta a_{\mathrm{w}}$. The observed deviations were both to higher and lower IN temperatures, depending on solute type and concentration. This finding is in accordance with Whale et al. (2018), who found an increase in IN activity for the $\mathrm{K}$-feldspars microcline and sanidine in dilute $\left(\mathrm{NH}_{4}\right)_{2} \mathrm{SO}_{4}$ solutions, but a decrease in the presence of dilute $\mathrm{NaCl}$.

In this paper and Part 3 (Kumar et al., 2019) of the companion papers, we relate IN activities of mineral surfaces more closely with the mineral surface properties by investigating the differences in IN activity of structurally similar minerals in pure water and aqueous solutions. In Part 3 we investigate the differences in IN activities of aluminosilicates and whether the enhancement observed for microcline 
in dilute $\mathrm{NH}_{3} / \mathrm{NH}_{4}^{+}$-containing solutions (shown in Part 1) is a more general property of aluminosilicates. In the current study, we present immersion freezing experiments of silicas (crystalline quartz and amorphous silicas) in pure water and in solution droplets in order to investigate their IN activity and how it is influenced by the presence of $\mathrm{NH}_{3}$ and several inorganic salts, namely $\left(\mathrm{NH}_{4}\right)_{2} \mathrm{SO}_{4}, \mathrm{NH}_{4} \mathrm{HSO}_{4}, \mathrm{Na}_{2} \mathrm{SO}_{4}$ and $\mathrm{NaOH}$. To elucidate which surface structures provide IN activity, we compare the IN activity of quartz with other silica particles, assuming that IN does not occur on the whole particle surface with a uniform probability but that the surface exhibits active sites, i.e., preferred locations for IN with areas of $10-50 \mathrm{~nm}^{2}$ based on estimates using classical nucleation theory (Vali, 2014; Vali et al., 2015; Kaufmann et al., 2017).

Quartz is one of the most abundant minerals in the Earth's crust. Since quartz is of relevance to different scientific fields such as material sciences, geochemistry and chemical engineering, quartz surface properties and processes have been the subject of many scientific studies. The dissolution and crystallization of quartz has gained great attention because it influences geochemical processes, such as the formation of mineralized deposits or the silica concentration in natural and industrial waters (Crundwell, 2017). Dry applications of quartz powders is of concern because of the pathogenicity of the ground particles (Fubini et al., 1989). We make use of the detailed characterization resulting from such studies to relate the IN activity of quartz to its surface structure.

\section{Methodology}

\subsection{Mineralogy, size distribution, milling and BET surface area measurements}

Mineralogy. Silica (three-dimensional polymeric network of $\mathrm{SiO}_{2}$ ) can exist in many different forms that can be crystalline or amorphous. Amorphous silica shows only shortrange order and lacks a crystalline structure as shown via Xray diffraction (XRD) measurements (Poulsen et al., 1995). Quartz, the most common form of crystalline silica, has a continuous regular framework of tetrahedral $\mathrm{SiO}_{4}$ units with $\mathrm{Si}$ in the center and oxygen atoms at the tetrahedral corners. Each oxygen atom is shared between two tetrahedra. It is a hard mineral (Mohs hardness 7) with no preferred cleavage plane (owing to the roughly equal bond strengths throughout the crystal structure) and typically breaks with conchoidal fracture. Quartz is the last mineral to crystallize from a magma, i.e., it crystallizes at lower temperatures compared to other minerals, and therefore it grows to fill the spaces remaining between the other crystals in the form of a common impurity (Bowen, 1922, 1928).

Size distribution. Quartz from Sigma-Aldrich ( 99\%) was the primary sample used in this study (see Sect. 4.4.5 for a discussion of the $1 \%$ of impurities). We will refer to this sample in the following as SA quartz. As per manufac- turer, SA quartz is a naturally occurring microcrystalline silica that has been finely ground resulting in a particle size range of $0.5-10 \mu \mathrm{m}$ (approx. $80 \%$ between $1-5 \mu \mathrm{m}$ ). In addition, we determined the number size distribution with a TSI 3080 scanning mobility particle sizer (SMPS) and a TSI 3321 aerodynamic particle sizer (APS). Two lognormal distributions were fitted to the bimodal size distribution yielding mode diameters of $482 \mathrm{~nm}$ and $1.52 \mu \mathrm{m}$ (see the Supplement). Scanning electron microscopy (SEM) measurements were also performed on the quartz sample at the ScopeM facility at ETH Zurich. The quartz samples were prepared by placing them on a graphite plate and coated with $\mathrm{Pt} / \mathrm{Pd}$ alloy by sputtering before taking the images (see the Supplement). SEM measurements mostly concur to the size range provided by the manufacturer with the exception of the presence of very few larger particles ( $>10 \mu \mathrm{m}$ in diameter), which are not measured by APS since they are not well aerosolized owing to their higher mass. We also determined the mineralogical composition of the SA quartz sample by means of XRD in order to assess the mineralogical purity of the mineral. Rietveld refinement using Profex software (Döbelin and Kleeberg, 2015) was performed for a quantitative analysis. Based on the X-ray diffractogram, the sample of SA quartz consists of $98.9 \%( \pm 0.29 \%)$ quartz, mixed with kaolinite $(0.32 \% \pm 0.2 \%)$ and topaz $(0.76 \% \pm 0.2 \%)$. The amorphous content is estimated as $4.5 \pm 0.5 \%$. The Brunauer-EmmettTeller (BET) nitrogen adsorption method was used to determine the specific surface area of this quartz sample as $4.91 \mathrm{~m}^{2} \mathrm{~g}^{-1}$. In addition, thermogravimetric analysis (TGA) was performed on the quartz sample to assess the presence of volatile species. The $0.30 \%$ loss in weight was observed in TGA up to $350^{\circ} \mathrm{C}$ (see the Supplement).

Additional milling. To assess the effect of further milling on the IN efficiency of the SA quartz sample, we milled a portion of the sample with a tungsten carbide disc mill for $40 \mathrm{~s}$ (with 1 min gap after the first $20 \mathrm{~s}$ of milling) before running emulsion freezing experiments.

Other samples. For comparison, we also used four other silica dusts besides SA quartz:

a. The quartz sample (BET value $3.67 \mathrm{~m}^{2} \mathrm{~g}^{-1}$ ) that showed little IN activity in Kaufmann et al. (2016) (termed Kaufmann quartz) (see Appendix A for details and explanation for its previously reported low IN activity). The $0.17 \%$ loss in weight was observed in TGA up to $350^{\circ} \mathrm{C}$ (see the Supplement). The amorphous content is estimated as $6.4 \pm 0.5 \%$.

b. A crystalline quartz sample procured from the Technical University of Vienna (termed TU Vienna quartz), which is "quartz I" of Zolles et al. (2015). The $0.20 \%$ loss in weight was observed in TGA up to $350{ }^{\circ} \mathrm{C}$ (see the Supplement).

c. Amorphous silica particles procured from Alfa Aesar (particle size: 0.4-0.6 $\mu \mathrm{m}$ (characterization by manufac- 
turer), BET: $5.72 \mathrm{~m}^{2} \mathrm{~g}^{-1}$ ). In order to assess the effect of milling on the IN efficiency of amorphous silica, we milled a portion of this sample with a tungsten carbide disc mill for $40 \mathrm{~s}$ (with 1 min gap after the first $20 \mathrm{~s}$ of milling).

d. Nonporous, amorphous silica particles procured from Zurich University of Applied Sciences (named Stöber particles in this article, mean particle diameter: $0.34 \pm$ $0.02 \mu \mathrm{m}$, BET: $11 \mathrm{~m}^{2} \mathrm{~g}^{-1}$, see the Supplement for synthesis procedure).

Please note that both Alfa Aesar and Stöber silica particles are synthetically grown (using tetraethyl orthosilicate, TEOS, in alkaline conditions) samples used in this study and have not been milled. Only when indicated we milled the Alfa Aesar silica particles on purpose.

\subsection{Emulsion freezing of quartz freshly suspended in pure water or solutions}

Immersion freezing experiments were carried out with the differential scanning calorimeter (DSC) setup (Q10 from TA Instruments). The $5 \mathrm{wt} \%$ SA quartz suspensions in water (molecular biology reagent water from Sigma-Aldrich) were prepared in borosilicate glass vials with varying solute concentrations $(0 \mathrm{wt} \%-20 \mathrm{wt} \%)$ viz. $\left(\mathrm{NH}_{4}\right)_{2} \mathrm{SO}_{4}$ (SigmaAldrich, $\geq 99 \%$ ), $\mathrm{NH}_{4} \mathrm{HSO}_{4}$ (Sigma-Aldrich, $\geq 99.5 \%$ ), $\mathrm{Na}_{2} \mathrm{SO}_{4}$ (Sigma-Aldrich, $\geq 99 \%$ ), $\mathrm{NaOH}$ (Fluka Chemical, $\geq 99 \%$ ), $\mathrm{NH}_{3}$ solution (Merck, $25 \%$ ). For comparison, $5 \mathrm{wt} \%$ TU Vienna quartz and a concentrated ( $8 \mathrm{wt} \%-9 \mathrm{wt} \%)$ Kaufmann quartz sample in water, as well as $10 \mathrm{wt} \%$ Stöber silica and Alfa Aesar silica suspensions in water containing $\mathrm{NH}_{3}$ and $\left(\mathrm{NH}_{4}\right)_{2} \mathrm{SO}_{4}$, were also prepared for freezing experiments (See Appendix A for freezing experiment details for the Kaufmann quartz sample).

To avoid particle aggregation, the suspensions prepared in pure water or solutions were sonicated for $5 \mathrm{~min}$ before preparing the emulsions. The aqueous suspension and an oil/surfactant mixture (95 wt \% mineral oil, Sigma-Aldrich, and $5 \mathrm{wt} \%$ lanolin, Fluka Chemical) were mixed in a ratio of $1: 4$ and emulsified with a rotor-stator homogenizer (Polytron PT 1300D with a PT-DA 1307/2EC dispersing aggregate) for $40 \mathrm{~s}$ at $7000 \mathrm{rpm}$. This procedure leads to droplet size distributions peaking at about $2-3 \mu \mathrm{m}$ in number and a broad distribution in volume with highest values between 4 and $12 \mu \mathrm{m}$ similar to the size distributions shown in Fig. 1 of Marcolli et al. (2007), Pinti et al. (2012) and Kaufmann et al. (2016). Regular inspection under the microscope did not reveal any effect of dust particles or solutes on the droplet size distribution. We placed 4-8 $\mathrm{mg}$ of this emulsion in an aluminum pan, which was hermetically closed and subjected to three freezing cycles in the DSC following the method developed and described by Marcolli et al. (2007). The first and the third freezing cycles were executed at a cooling rate of $10 \mathrm{~K} \mathrm{~min}^{-1}$ to control the stability of the emulsion. The

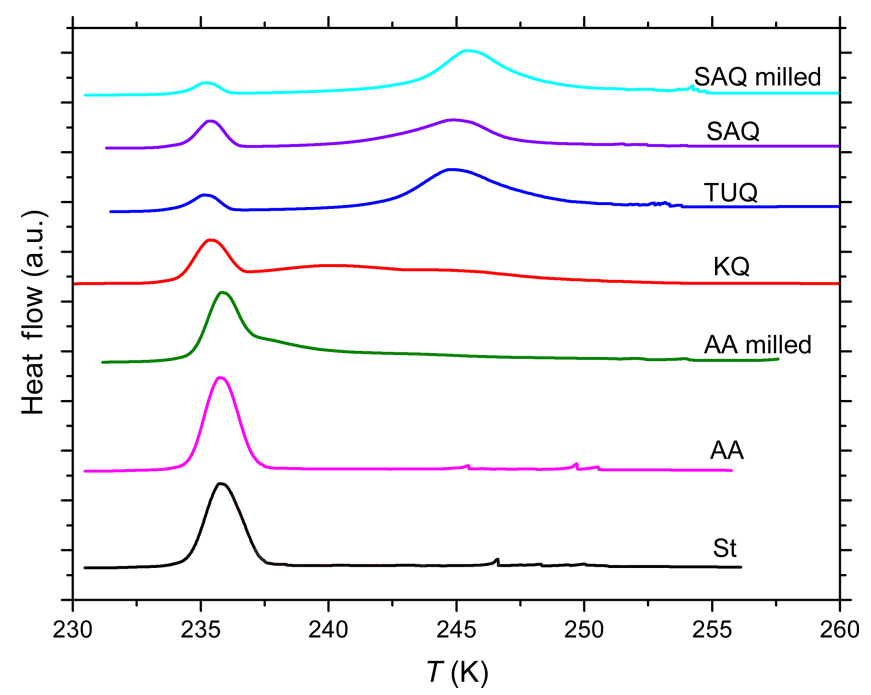

Figure 1. DSC thermograms of various quartz and amorphous silica particles suspended in pure water. St: Stöber silica $(10 \mathrm{wt} \%)$; AA: Alfa Aesar silica (10 wt \%); AA milled: milled Alfa Aesar silica (10 wt \%); KQ: quartz sample from Kaufmann et al. (2016) (8 wt \%-9 wt \%); TUQ: TU Vienna quartz (5 wt \%); SAQ: SigmaAldrich quartz (5 wt \%, milled by Sigma-Aldrich); SAQ milled: additionally milled Sigma-Aldrich quartz (5 wt \%). All curves are normalized such that the total areas under the heterogeneous plus homogeneous freezing curves sum up to the same value.

second freezing cycle was run at $1 \mathrm{~K} \mathrm{~min}^{-1}$ cooling rate and used for evaluation (Zobrist et al., 2008; Pinti et al., 2012; Kaufmann et al., 2016; Kumar et al., 2018a).

The DSC registers the heat release when emulsion droplets freeze. When emulsions are prepared from an aqueous suspension of INPs, the larger droplets are expected to freeze heterogeneously because they contain many particles while the smaller ones rather freeze homogeneously because they contain only few or no particles. Typical DSC thermograms therefore contain a freezing peak below about $237 \mathrm{~K}$ due to homogeneous IN, while freezing above this temperature is due to heterogeneous IN. The onset temperatures of the heterogeneous freezing peak $\left(T_{\text {het }}\right)$ and the homogeneous freezing peak $\left(T_{\text {hom }}\right)$ were determined as the intersection of the tangent drawn at the point of greatest slope at the leading edge of the peak with the extrapolated baseline, whereas the melting temperature $\left(T_{\text {melt }}\right)$ was determined as the maximum of the ice melting peak (see Fig. 1 of Kumar et al., 2018a). The heat release is approximately proportional to the volume of water that froze heterogeneously or homogeneously and is represented by the integral of the peak over time. Note that this proportionality is only approximate because the enthalpy of freezing exhibits a temperature dependence (Speedy, 1987; Johari et al., 1994). We quantified the heterogeneously frozen fraction, $F_{\text {het }}$, as the ratio of the heterogeneous freezing signal to the total freezing signal of the thermogram in the time domain (see Kumar et al., 2018a for 
details). The evaluation of $F_{\text {het }}$ does not include the spikes that occur before the appearance of the heterogeneous freezing signal. These spikes originate from single droplets in the tail of the droplet size distribution (mostly between 100$300 \mu \mathrm{m}$ with some up to $500 \mu \mathrm{m}$ in diameter) being orders of magnitude larger in volume than the average droplets and not representative for the sample. Freezing experiments were performed on emulsions prepared from at least two separate quartz suspensions for each solute concentration and means are reported. Average precisions in $T_{\text {het }}$ are $\pm 0.2 \mathrm{~K}$ with maximum deviations not exceeding $0.9 \mathrm{~K}$ (i.e., uncertainties slightly higher than in Kumar et al., 2018a). $T_{\text {hom }}$ and $T_{\text {melt }}$ are precise within $\pm 0.1 \mathrm{~K}$. Absolute uncertainties in $F_{\text {het }}$ are on average \pm 0.02 and do not exceed \pm 0.12 . However, $F_{\text {het }}$ carries larger uncertainties (up to \pm 0.19 ) when heterogeneous freezing signals are weak or overlap (forming a shoulder) with the homogeneous freezing signal (as was the case for amorphous silica in various solutions and quartz aged in $\mathrm{NaOH}$ solution).

\subsection{Aging and reversibility of quartz suspended in water or solutions}

SA quartz $(5 \mathrm{wt} \%)$ suspended in pure water, $\mathrm{NH}_{3}$ solution (0.005 molal; $\left.\mathrm{pH} 7.9 ; a_{\mathrm{w}} \approx 0.999\right),\left(\mathrm{NH}_{4}\right)_{2} \mathrm{SO}_{4}$ solution (10 wt \%; pH 5.5; $\left.a_{\mathrm{w}} \approx 0.963\right), \mathrm{NH}_{4} \mathrm{HSO}_{4}$ solution ( $2 \mathrm{wt} \%$; $\left.\mathrm{pH} 1.1 ; a_{\mathrm{w}} \approx 0.988\right), \mathrm{Na}_{2} \mathrm{SO}_{4}$ solution $\left(5 \mathrm{wt} \% ; \mathrm{pH} 6.8 ; a_{\mathrm{w}} \approx\right.$ $0.986)$ or $\mathrm{NaOH}$ solution $\left(5 \times 10^{-3}\right.$ molal; $\mathrm{pH} 9.5 ; a_{\mathrm{w}} \approx 1$ and $5 \times 10^{-6}$ molal; $\mathrm{pH} 7.1 ; a_{\mathrm{w}} \approx 1$ ) were aged in borosilicate glass vials and tested over a period of 5 days. Immersion freezing experiments were carried out with the DSC setup with emulsions prepared from at least two separate aging experiments for each solute concentration. Measurements were done on the day of preparation (fresh) and on the subsequent 5 days in order to assess the evolution and long-term effect of these solutes on the IN efficiency of quartz. Aging experiments conducted in pure water, $\mathrm{NH}_{3}$ solution $(0.005$ molal) and $\mathrm{NaOH}$ solution $\left(5 \times 10^{-3}\right.$ molal $)$ were repeated in polypropylene Falcon tubes to assess the influence of leached contaminants from the borosilicate glass vials on the IN activity of quartz.

After aging for 5 days the suspensions were centrifuged for $2 \mathrm{~min}$ at $600 \mathrm{rpm}$, the supernatant solution was removed and the settled particles were washed with pure water. This process was repeated five times and the washed particles were resuspended in pure water and the IN efficiency of emulsions prepared from these suspensions were tested with the DSC setup.

In order to assess the leaching of contaminants from quartz and the vial walls, suspensions of quartz were prepared in pure water in both borosilicate glass vials and polypropylene Falcon tubes and aged for $72 \mathrm{~h}$. The freshly prepared and aged suspensions were centrifuged to remove the particles. The supernatant liquid in each case was collected and tested for the concentration of leached elements via induc- tively coupled plasma mass spectroscopy (ICP-MS). The results of ICP-MS measurements are summarized in the Supplement (Sect. S10).

\section{Results}

\subsection{Ice nucleation activity of quartz and amorphous silica in pure water}

Figure 1 shows the DSC thermograms of suspensions of quartz and amorphous silica particles in pure water prepared as emulsion droplets. $F_{\text {het }}$ and $T_{\text {het }}$ are listed in Table 1 . As can be easily seen, there are large differences in IN activities between the samples, from barely to highly IN active.

\subsubsection{Quartz}

In the emulsion freezing experiments, all investigated quartz samples clearly show IN activity. TU Vienna quartz is slightly more IN active (with respect to both $T_{\text {het }}$ and $F_{\text {het }}$ ) than SA and Kaufmann quartz at similar suspension concentrations. $T_{\text {het }}$ of TU Vienna quartz $(250.9 \mathrm{~K})$ is slightly lower than the freezing onset reported by Zolles et al. (2015) $(\sim 252 \mathrm{~K})$. We ascribe this difference to the higher quartz surface area present in the Zolles et al. (2015) droplet freezing setup compared to our emulsion freezing experiments. In accordance with Zolles et al. (2015), we observe an enhancement in IN efficiency of quartz due to milling (Table 1).

The quartz sample from Kaufmann et al. (2016) is clearly IN active, exhibiting a distinct heterogeneous freezing signal with $T_{\text {het }} \approx 242 \mathrm{~K}$ and a shoulder extending to higher temperature with $T_{\text {het }} \approx 250 \mathrm{~K}$. Kaufmann et al. (2016) reported a heterogeneous freezing onset temperature of $\sim 247 \mathrm{~K}$ for the same sample, yet with a very weak heterogeneous freezing signal corresponding to an IN active particle fraction of only 0.01. We explain in Appendix A that this was due to an underestimation of the coarse particle fraction because of the presence of very large particles $(>20 \mu \mathrm{m})$, which were not accounted for in the particle size distribution determined by SMPS/APS leading to a bias resulting in a too low estimate of the IN active fraction of quartz particles.

\subsubsection{Amorphous silica particles}

DSC thermograms of both amorphous silica samples (Fig. 1) show only a single freezing peak with onsets of 237.2 and 237.1 K for Stöber and Alfa Aesar silica, respectively, listed under $T_{\text {het }}$ in Table 1 . This freezing temperature is slightly higher but still within the uncertainty range of $T_{\text {hom }}$ of pure water emulsions (i.e., $237.0 \mathrm{~K}$ ). Note that due to the volume dependence of homogeneous IN rates, $T_{\text {hom }}$ of the quartz samples is slightly lower than $T_{\text {hom }}$ of the pure water emulsions. Only the smallest droplets of the emulsified quartz suspensions are empty and therefore freeze homogeneously at a lower temperature than the larger droplets that give rise to the 
Table 1. Comparison of IN efficiency (in pure water) of various quartz and amorphous silica particles based on emulsion freezing experiments.

\begin{tabular}{lrrrr}
\hline Sample name & $\begin{array}{r}\text { Suspension } \\
\text { concentration } \\
(\text { wt } \%)\end{array}$ & $T_{\text {hom }}(\mathrm{K})$ & $T_{\text {het }}(\mathrm{K})$ & $F_{\text {het }}$ \\
& 10 & $237.0^{\mathrm{a}}$ & $237.2^{\mathrm{b}}$ & 0.05 \\
Stöber & 10 & $237.0^{\mathrm{a}}$ & $237.1^{\mathrm{b}}$ & 0.03 \\
Alfa Aesar & 10 & 236.8 & $246.8 / 239.7^{\mathrm{c}}$ & 0.42 \\
Alfa Aesar (milled) & $8-9$ & 236.5 & $250.2 / 241.8^{\mathrm{c}}$ & 0.70 \\
Quartz (Kaufmann et al., 2016) & 5 & 236.6 & 250.9 & 0.87 \\
TU Vienna $_{\text {Sigma-Aldrich }}^{\text {d }}$ & 5 & 236.5 & 247.6 & 0.82 \\
Sigma-Aldrich $^{\text {d }}$ & 1 & 236.7 & 246.9 & 0.68 \\
Sigma-Aldrich (milled) $^{\mathrm{e}}$ & 5 & 236.4 & 249.0 & 0.92 \\
Sigma-Aldrich (milled) $^{\mathrm{e}}$ & 1 & 236.5 & 247.4 & 0.79 \\
\hline
\end{tabular}

${ }^{\text {a }}$ Mean $T_{\text {hom }}$ of pure water emulsions is reported here since the onset of the homogeneous freezing signal cannot be separated from the presumed heterogeneous freezing signal. ${ }^{\mathrm{b}}$ Onsets of heterogeneous and homogeneous freezing signals are nearly indistinguishable. The observed onset lies within the precision range of $T_{\mathrm{hom}}(237.0 \mathrm{~K}$, taken as the point dividing heterogeneous and homogeneous freezing signal to evaluate $F_{\text {het }}$ ). ${ }^{\mathrm{c}}$ Onsets of the two shoulders exhibited by these samples (see Fig. 1). $F_{\text {het }}$ is based on the whole heterogeneous freezing signal.

${ }^{d}$ Milled quartz as obtained from Sigma-Aldrich. ${ }^{\mathrm{e}}$ Additionally milled Sigma-Aldrich quartz.

onset of the homogeneous freezing peak in pure water emulsions. The calculation of the heterogeneously frozen fraction for both amorphous silica samples assumes that the heat signal at $T>237.0 \mathrm{~K}$ is due to heterogeneous freezing, leading to $F_{\text {het }}$ below the uncertainty limits (see Table 1 ). Since both samples consist of submicron particles that should be well distributed between emulsion droplets (droplet size needed to incorporate on average one silica particle is $\sim 1.1 \mu \mathrm{m}$ in diameter), a prevalence of empty droplets cannot be the reason of the absence of detectable IN activity. Therefore, based on our emulsion freezing experiments we consider the amorphous silica particles as inactive or barely IN inactive in water.

When the Alfa Aesar sample is milled, a clearly visible heterogeneous freezing signal develops consisting of two shoulders on the warmer end of the homogeneous freezing peak with onsets of 246.8 and $239.7 \mathrm{~K}$, again demonstrating the fundamental importance of the milling process.

\subsection{Dependence of the heterogeneous freezing temperatures on the presence of solutes}

For freezing experiments in the presence of solutes we concentrate on the SA quartz. The mean heterogeneous freezing onset $\left(T_{\text {het }}\right)$, homogeneous freezing onset $\left(T_{\text {hom }}\right)$ and ice melting temperatures $\left(T_{\text {melt }}\right)$ for $5 \mathrm{wt} \% \mathrm{SA}$ quartz suspensions in water and aqueous solution droplets are shown in Fig. $2 \mathrm{a}$ as a function of the solution water activity $\left(a_{\mathrm{w}}\right)$. The $a_{\mathrm{w}}$ is obtained from the evaluation of the melting point depression measured during the heating cycle using the Koop et al. (2000) parameterization. Hence all melting temperatures lie exactly on the melting curve, except in the case of $\mathrm{Na}_{2} \mathrm{SO}_{4}$ where above the eutectic concentration of $4.6 \mathrm{wt} \%$ a hydrate of $\mathrm{Na}_{2} \mathrm{SO}_{4}$ crystallizes together with ice and $a_{\mathrm{w}}$ had to be calculated based on the solute concentration using the AIOMFAC thermodynamic model at $298 \mathrm{~K}$ (Zuend et al., 2008, 2011). The measured $T_{\text {hom }}$ follows a similar $a_{\mathrm{w}}$ dependency as $T_{\text {melt }}$ and has been parameterized by Koop et al. (2000). We construct this line by a constant shift of the melting curve by $\Delta a_{\mathrm{w}}^{\text {hom }}(T)=0.30$ (dotted black line) derived using the averaged $T_{\text {hom }}$ of all experiments of this study (which is in good agreement with $\Delta a_{\mathrm{w}}^{\text {hom }}(T)=0.305$ reported by Koop et al., 2000). Similarly, we apply a constant offset $\Delta a_{\mathrm{w}}^{\text {het }}=0.221$ to shift the ice melting curve to the heterogeneous freezing temperature of pure water, yielding the solid black line, which for simplicity will be referred to as $T_{\text {het }}^{\Delta a_{\mathrm{w}}^{\text {het }}}\left(a_{\mathrm{w}}\right)$ from here onwards (see Kumar et al., 2018a for more details).

Analogous to the parameterization for $\Delta a_{\mathrm{w}}^{\text {hom }}(T)$ based on the thermodynamic homogeneous IN description of Koop et al. (2000), $T_{\text {het }}^{\Delta a_{\mathrm{w}}^{\text {het }}}\left(a_{\mathrm{w}}\right)$ assumes that the water activity dependence of $T_{\text {het }}$ is determined by solute-driven changes in the structure of the water alone, while interactions of the solute with the INP surface are excluded. From Kumar et al. (2018a, 2019) we know that the assumption of $\Delta a_{\mathrm{w}}^{\text {het }}$ fails when there are specific interactions between the solute and the mineral surface. As can be seen from Fig. 2a the measured heterogeneous freezing onset temperatures, $T_{\text {het }}$, follow $T_{\text {het }}^{\Delta a_{\mathrm{W}}^{\text {het }}}\left(a_{\mathrm{W}}\right)$ within measurement uncertainties for quartz suspensions in $\left(\mathrm{NH}_{4}\right)_{2} \mathrm{SO}_{4}, \mathrm{NH}_{4} \mathrm{HSO}_{4}$ and $\mathrm{Na}_{2} \mathrm{SO}_{4}$, but fall below this line in the presence of the bases $\mathrm{NH}_{3}$ and $\mathrm{NaOH}$. In these alkaline solutions, $T_{\text {het }}$ for quartz emulsions strongly falls below $T_{\text {het }}^{\Delta a_{\mathrm{w}}^{\text {het }}}\left(a_{\mathrm{w}}\right)$ at dilute solute concentrations $\left(a_{\mathrm{w}} \geq\right.$ $0.99)$ and stay almost parallel to $T_{\text {het }}^{\Delta a_{\mathrm{w}}^{\text {het }}}\left(a_{\mathrm{w}}\right)$ to higher solute concentrations. This decrease in $T_{\text {het }}$ is less pronounced in the presence of $\mathrm{NH}_{3}$ than for suspensions in $\mathrm{NaOH}$. 

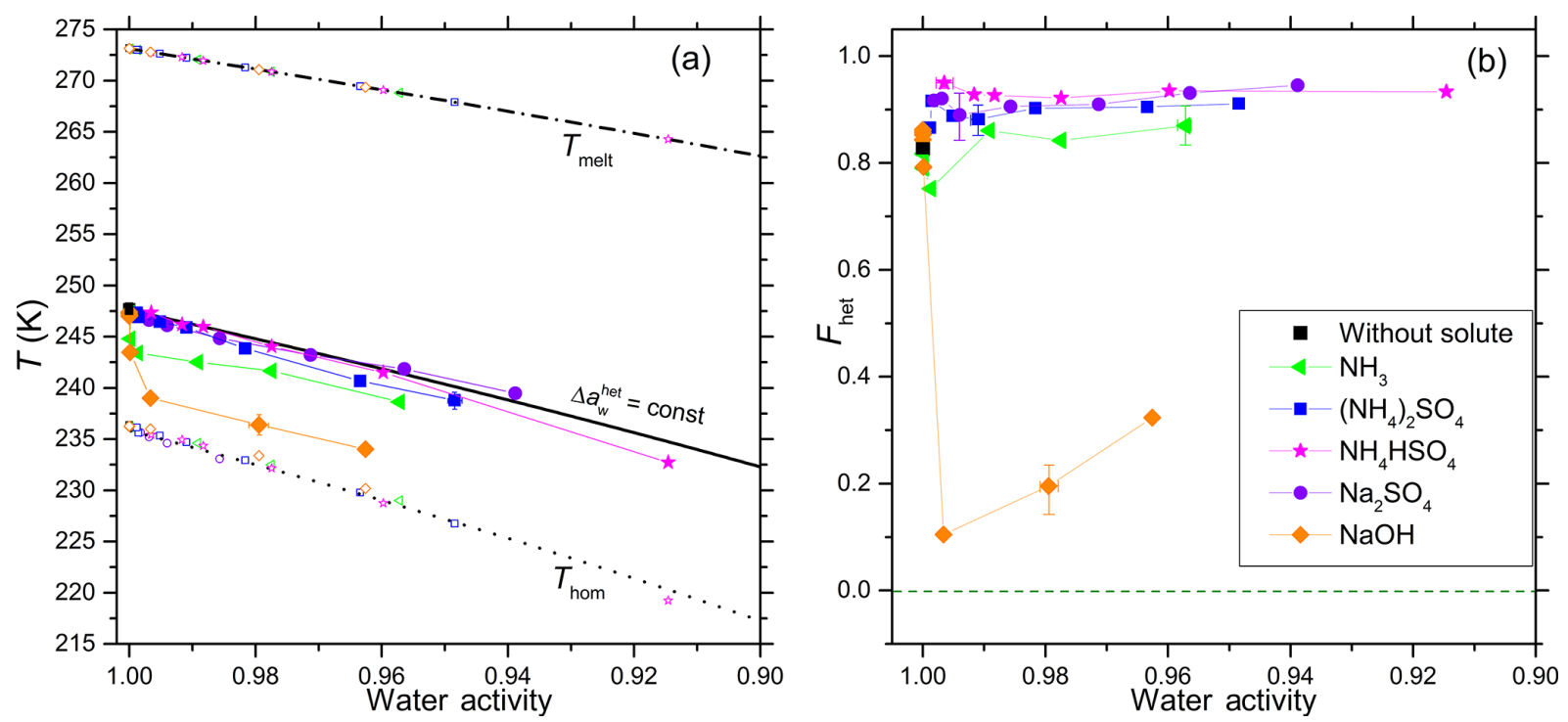

Figure 2. (a) Onset freezing temperatures of emulsion freezing experiments performed with $5 \mathrm{wt} \%$ SA quartz suspended in aqueous solutions of inorganic solutes (for symbols and colors see insert). Heterogeneous freezing onset temperatures, $T_{\text {het }}$ (filled solid symbols connected by thin lines); homogeneous freezing onset temperatures, $T_{\text {hom }}$ (open symbols at $T<237 \mathrm{~K}$ ), and ice melting temperatures, $T_{\text {melt }}$ (open symbols at $T>262 \mathrm{~K}$ ) are given as functions of the solution's water activity, $a_{\mathrm{w}}$. Dash-dotted black line: ice melting curve. Dotted black line: homogeneous ice freezing curve for supercooled aqueous solutions obtained by horizontally shifting the ice melting curve by a constant offset $\Delta a_{\mathrm{w}}^{\text {hom }}(T)=0.30$. Solid black line: horizontally shifted from the ice melting curve by $\Delta a_{\mathrm{w}}^{\text {het }}(T)=0.221$ derived from the heterogeneous freezing temperature of the suspension of quartz in pure water (filled black square at $a_{\mathrm{w}}=1$ ). Symbols are the mean of at least two emulsion freezing experiments (using at least two separate suspensions). Two symbols carry error bars to show representative experimental variations (min to max) in $T_{\text {het }}$ and $a_{\mathrm{w}}$. (b) Heterogeneously frozen fraction $F_{\text {het }}$ as a function of the solution's water activity $\left(a_{\mathrm{W}}\right)$. Five symbols carry error bars showing representative experimental variations ( $\min$ to max) in $F_{\text {het }}$ and $a_{\mathrm{w}}$. Absolute uncertainties in $F_{\text {het }}$ do not exceed \pm 0.12 .

\subsection{Dependence of the heterogeneously frozen fraction on the presence of solutes}

While the addition of neutral or acidic solutes does not influence the freezing temperature beyond the expected freezing point depression described by $T_{\mathrm{het}}^{\Delta a_{\mathrm{w}}^{\text {het }}}\left(a_{\mathrm{w}}\right)$, it does affect the heterogeneously frozen fraction. Figure 3 shows the DSC thermograms for emulsion freezing of $5 \mathrm{wt} \% \mathrm{SA}$ quartz suspended in increasingly concentrated $\left(\mathrm{NH}_{4}\right)_{2} \mathrm{SO}_{4}$ solutions. The dotted brown line connecting the onsets of the heterogeneous freezing signals depicts the continuous decrease in $T_{\text {het }}$ as the $\left(\mathrm{NH}_{4}\right)_{2} \mathrm{SO}_{4}$ concentration increases. An increase in heterogeneous-to-homogeneous freezing ratio with increasing solute concentration is apparent. $F_{\text {het }}$ increases up to $a_{\mathrm{w}}=0.998(0.5 \mathrm{wt} \%)$ and stays around this increased value when the solute concentration is further increased. Figure $2 b$ shows the evaluation of the freezing signals in terms of the heterogeneously frozen fraction, $F_{\text {het }}$, as a function of $a_{\mathrm{w}}$ for all investigated solutes. For solutions containing the salts $\left(\mathrm{NH}_{4}\right)_{2} \mathrm{SO}_{4}, \mathrm{NH}_{4} \mathrm{HSO}_{4}$ and $\mathrm{Na}_{2} \mathrm{SO}_{4}, F_{\text {het }}$ shows a constant increase compared with the pure water case, albeit hardly exceeding the maximum uncertainty limit. Despite the decrease in $T_{\text {het }}$, there seems to be a slight increase in $F_{\text {het }}$ for quartz suspended in aqueous solutions with higher $\mathrm{NH}_{3}\left(a_{\mathrm{w}} \geq 0.98\right)$ concentrations. On the other hand, even very low concentra- tions of $\mathrm{NaOH}\left(a_{\mathrm{w}} \leq 0.99\right)$ strongly decrease $F_{\text {het }}$. The heterogeneously frozen fraction slightly recovers at higher concentrations of $\mathrm{NaOH}\left(a_{\mathrm{w}}=0.99-0.96\right)$ yet remains significantly below the pure water case.

Furthermore, in Part 3 (Kumar et al., 2019) we show that micas (muscovite and biotite) and gibbsite, which reveal no IN activity in pure water emulsion freezing experiments, develop a heterogeneous freezing signal in the presence of $\mathrm{NH}_{3}$ and $\left(\mathrm{NH}_{4}\right)_{2} \mathrm{SO}_{4}$. We therefore tested the amorphous silica samples for a similar effect. DSC thermograms of both amorphous silica samples suspended in $\mathrm{NH}_{3}(0.05$ and 0.5 molal) and $\left(\mathrm{NH}_{4}\right)_{2} \mathrm{SO}_{4}(0.05 \mathrm{wt} \%$ and $1 \mathrm{wt} \%)$ solutions, corresponding to an $a_{\mathrm{w}}$ range of 1-0.987 (Fig. 4), show only one clear freezing signal. We report the onset of this freezing peak as $T_{\text {het }}$ in Table 2, while under $T_{\text {hom }}$ we list the onset freezing temperature of the reference solutions (prepared with the solute only). Neither the Alfa Aesar nor the Stöber silica particles (10 wt \% suspensions) show a significant increase in the freezing onset compared with the reference measurements. We evaluate the heterogeneously frozen fraction by attributing the freezing signal at temperatures above the reported $T_{\text {hom }}$ of the reference measurement to heterogeneous freezing, yielding $F_{\text {het }}$ within the uncertainty range (see Table 2). We therefore conclude that $\mathrm{NH}_{3}$ and 


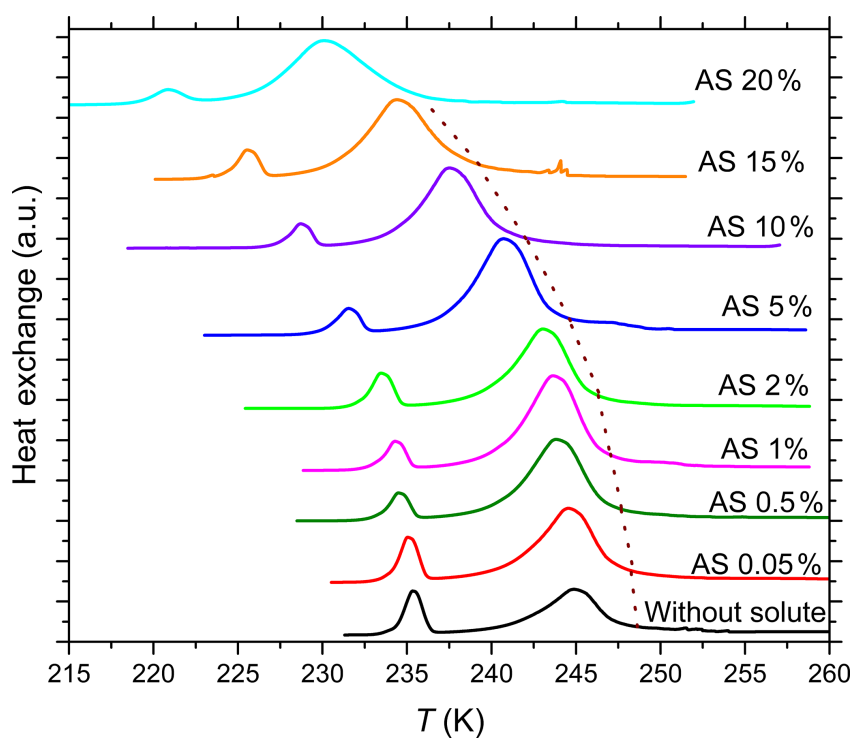

Figure 3. DSC thermograms of $5 \mathrm{wt} \%$ SA quartz particles suspended in ammonium sulfate (AS) solution droplets of increasing concentrations $(0 \mathrm{wt} \%-20 \mathrm{wt} \%$ AS). All curves are normalized such that the areas under the heterogeneous and homogeneous freezing curves sum up to the same value. The dotted brown line connects the heterogeneous freezing onset temperatures $\left(T_{\text {het }}\right)$ of the emulsions. With increasing AS concentration $T_{\text {het }}$ decreases monotonically while the intensity of the heterogeneous freezing signal increases initially in dilute AS solution and remains high up to high solute concentrations.

$\left(\mathrm{NH}_{4}\right)_{2} \mathrm{SO}_{4}$ do not lead to a discernable enhancement of the IN activity of amorphous silica.

\subsection{Aging and recovery experiments of quartz in water and aqueous solutions}

In order to assess the long-term effect of solutes on the IN efficiency of quartz, aging experiments were performed over a period of 5 days with $5 \mathrm{wt} \%$ SA quartz suspensions in pure water (prepared in borosilicate glass vials and polypropylene Falcon tubes) and various inorganic solutes. Every day, aliquots were taken from the suspension and tested in emulsion freezing experiments. For these experiments, $T_{\text {het }}$ and $F_{\text {het }}$ are given in the upper and lower panels of Figs. 5 and 6, respectively. Figure 5 shows the results of experiments performed in glass vials while Fig. 6 compares $T_{\text {het }}$ and $F_{\text {het }}$ of experiments performed in glass vials and polyprolylene tubes. After aging, the quartz suspensions were decanted, washed and resuspended in pure water in order to assess the reversibility of any surface modification occurring during the aging period. Figures 5 and 6 also show the change in $T_{\text {het }}$ and $F_{\text {het }}$ when aged quartz is resuspended in pure water. (Note that $T_{\text {het }}$ and $F_{\text {het }}$ in the experiments with fresh dusts can be lower than in the reversibility tests, because the former were performed in solutions and the latter in pure water.)

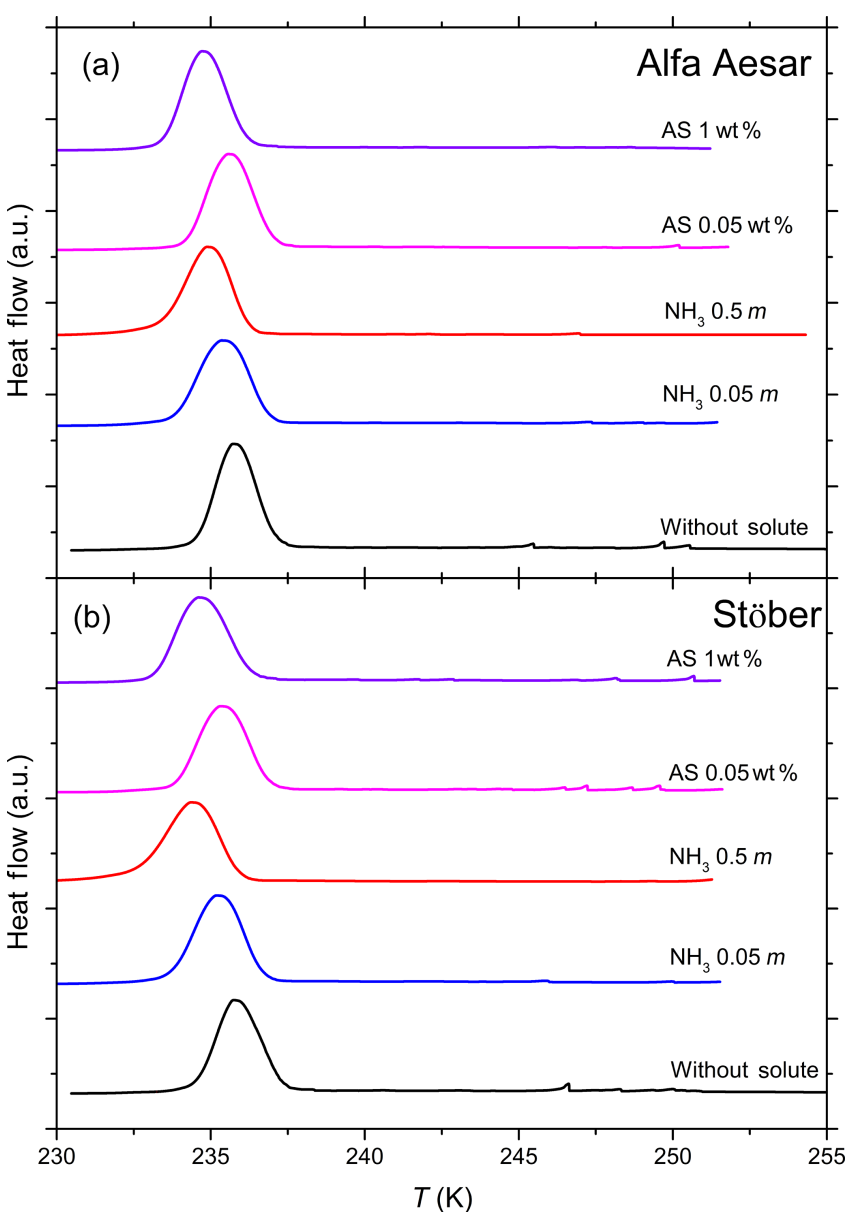

Figure 4. DSC thermograms of $10 \mathrm{wt} \%$ Alfa Aesar (a) and $10 \mathrm{wt} \%$ Stöber (b) silica particles suspended in pure water, ammonia $\left(\mathrm{NH}_{3}\right.$; 0.05 and 0.5 molal) and ammonium sulfate (AS; $0.05 \mathrm{wt} \%$ and $1 \mathrm{wt} \%$ ) solution droplets of varying concentrations (corresponding to $a_{\mathrm{w}}$ range of 1-0.987). All curves are normalized such that the areas under the heterogeneous and homogeneous freezing curves sum up to the same value. DSC thermograms of both amorphous silica samples show only one clear freezing signal that is indistinguishable from the homogeneous freezing signal. $T_{\text {hom }}$ of the corresponding emulsion freezing experiments with the pure solutions (in the absence of the silica particles) has been taken as the dividing temperature of heterogeneous and homogeneous freezing to evaluate $F_{\text {het }}$ (Table 2; see Sect. 3.3).

Aging of quartz suspensions in pure water in glass vials decreases the IN activity in terms of both $T_{\text {het }}$ and $F_{\text {het }}$. Strong decreases in IN efficiency occur during aging in the presence of $\mathrm{Na}_{2} \mathrm{SO}_{4}, \mathrm{NaOH}$ and $\mathrm{NH}_{3}$. On the other hand, $T_{\text {het }}$ and $F_{\text {het }}$ remain constant when quartz is suspended in $2 \mathrm{wt} \% \mathrm{NH}_{4} \mathrm{HSO}_{4}$ solution. Repetition of the aging experiments reveals considerable variability in the decrease in IN efficiency over time, as indicated by the large min-to-max bars after 2-5 days. The reversibility tests show complete or almost complete recovery of IN efficiency after washing in the case of aging in pure water and in solutions containing 
Table 2. Summary of the freezing experiments with emulsified aqueous solution droplets containing Alfa Aesar and Stöber amorphous silica particles $(10 \mathrm{wt} \%)$. Note that the absolute uncertainty in $F_{\text {het }}$ may be up to \pm 0.12 .

\begin{tabular}{lllrrrr}
\hline $\begin{array}{l}\text { Sample/suspension } \\
\text { concentration } \\
(\text { wt \% })\end{array}$ & Solute & $\begin{array}{l}\text { Solute } \\
\text { concentration } \\
\left(m^{\mathrm{a}} \text { or wt } \%\right)\end{array}$ & $a_{\mathrm{w}}$ & $T_{\text {hom }}(\mathrm{K})^{\mathrm{b}}$ & $T_{\text {het }}(\mathrm{K})$ & $F_{\text {het }}$ \\
\hline $\begin{array}{l}\text { Alfa Aesar } \\
(10 \mathrm{wt} \%)\end{array}$ & $\mathrm{NH}_{3}$ & $0.5 \mathrm{~m}$ & 0.987 & 236.3 & 236.5 & 0.01 \\
& $\mathrm{NH}_{3}$ & $0.05 \mathrm{~m}$ & 0.996 & 236.6 & 237.1 & 0.04 \\
& $\left(\mathrm{NH}_{4}\right)_{2} \mathrm{SO}_{4}$ & $1 \mathrm{wt} \%$ & 0.988 & 236.2 & 236.5 & 0.03 \\
& $\left(\mathrm{NH}_{4}\right)_{2} \mathrm{SO}_{4}$ & $0.05 \mathrm{wt} \%$ & 0.996 & 236.9 & 237.3 & 0.02 \\
& $\mathrm{Pure} \mathrm{water}$ & - & 1 & 237.0 & 237.1 & 0.03 \\
\hline Stöber & $\mathrm{NH}_{3}$ & $0.5 m$ & 0.997 & 236.3 & 236.2 & 0.01 \\
$(10 \mathrm{wt} \%)$ & $\mathrm{NH}_{3}$ & $0.05 m$ & 0.999 & 236.6 & 236.7 & 0.02 \\
& $\left(\mathrm{NH}_{4}\right)_{2} \mathrm{SO}_{4}$ & $1 \mathrm{wt} \%$ & 0.988 & 236.2 & 236.3 & 0.02 \\
& $\left(\mathrm{NH}_{4}\right)_{2} \mathrm{SO}_{4}$ & $0.05 \mathrm{wt} \%$ & 0.994 & 236.9 & 236.9 & 0.02 \\
& $\mathrm{Pure} \mathrm{water}$ & - & 1 & 237.0 & 237.2 & 0.05 \\
\hline
\end{tabular}

${ }^{\mathrm{a}} m=$ molality; ${ }^{\mathrm{b}}$ mean $T_{\text {hom }}$ of pure water/solution emulsions (taken as the point dividing heterogeneous and homogeneous freezing signal to evaluate $F_{\text {het }}$ ) is reported here since the onset of the homogeneous freezing signal cannot be separated from the presumed heterogeneous freezing signal.

$\mathrm{Na}_{2} \mathrm{SO}_{4}, \mathrm{NaOH}$ (except at high pH 9.5) and $\left(\mathrm{NH}_{4}\right)_{2} \mathrm{SO}_{4}$. Interestingly, instead of recovering, the IN efficiency after aging in a dilute $\mathrm{NH}_{3}$ solution decreases even further when the particles were washed and resuspended in pure water.

To elucidate whether leached material from the surface of the glass vial had any impact on the decrease in IN activity during aging of quartz in glass vials, experiments exhibiting a strong decrease in IN efficiency were repeated in polypropylene Falcon tubes. Indeed, in contrast to aging in glass vials, quartz aged in pure water in polypropylene tubes exhibited a stable $T_{\text {het }}$ and a slight enhancement in $F_{\text {het }}$ (yet within the uncertainty range) (Fig. 6). When quartz is suspended in a dilute $\mathrm{NH}_{3}$ (pH 7.9) solution in polypropylene tubes, similar trends were observed as in glass vials, with constant $T_{\text {het }}$ after an initial decrease, followed by a further decrease after washing with pure water. Interestingly, quartz freshly suspended in $\mathrm{NaOH}$ (pH 9.5) in polypropylene tubes shows a stronger initial decrease in IN efficiency ( $T_{\text {het }}$ and $F_{\text {het }}$ ) but stays higher from the second day onwards than in the corresponding experiments performed in a glass vial until the end of the experiment.

Another set of SA quartz suspensions ( $5 \mathrm{wt} \%)$ was prepared in pure water in glass vials and aged for 7 months to investigate the aging effect over very long timescales. Figure 7 shows that the aged particles almost completely lost their IN efficiency and barely any of it was recovered after the aged particles were washed and resuspended in pure water. In Sect. 4.4, we relate the results of the aging and reversibility experiments to surface processes occurring in the different solutions.

\section{Discussion}

\subsection{IN efficiency of quartz and amorphous silica particles in pure water}

As shown in Fig. 1 and Table 1, the IN activity of quartz is superior to the one of amorphous silica particles. The freezing onset temperatures in pure water for the investigated quartz samples range from 247 to $251 \mathrm{~K}$ (see Table 1), covering a similar temperature range as the quartz samples investigated by Atkinson et al. (2013) and Zolles et al. (2015). On the other hand, the IN activity of the silica particles (Stöber and Alfa Aesar) is negligible. Hardly any IN activity of amorphous silica particles is in agreement with Zobrist et al. (2008) who observed freezing at around $255 \mathrm{~K}$ in bulk freezing experiments with $3 \mu \mathrm{L}$ droplets containing $10^{9} \times 10^{10}$ particles (total mean particle surface area $0.25-2.5 \mathrm{~cm}^{2}$ ). This freezing temperature is close to the one of pure water droplets of this size $(252-253 \mathrm{~K})$. Whale et al. (2018) found IN active site densities of silica particles from Sigma-Aldrich (silica gel) of $n_{\mathrm{s}} \approx 10 \mathrm{~cm}^{-2}$ at $251 \mathrm{~K}$ and $n_{\mathrm{s}} \approx 0.1 \mathrm{~cm}^{-2}$ at $261 \mathrm{~K}$, corresponding to a slightly higher IN activity of these silica particles compared with those synthesized by Zobrist et al. (2008).

Zolles et al. (2015) investigated the density of IN active sites of three quartz samples and found a very high variability from hardly IN active to an activity similar to that of microcline. Their quartz sample from Sigma-Aldrich corresponding to the TU Vienna quartz sample in this study was the most IN active and a natural quartz sample the least IN active. Moreover, the IN activity of the natural quartz sample increased considerably upon milling. Our comparison of emulsion freezing experiments with SA quartz additionally milled and original SA quartz (used as obtained from the manufac- 


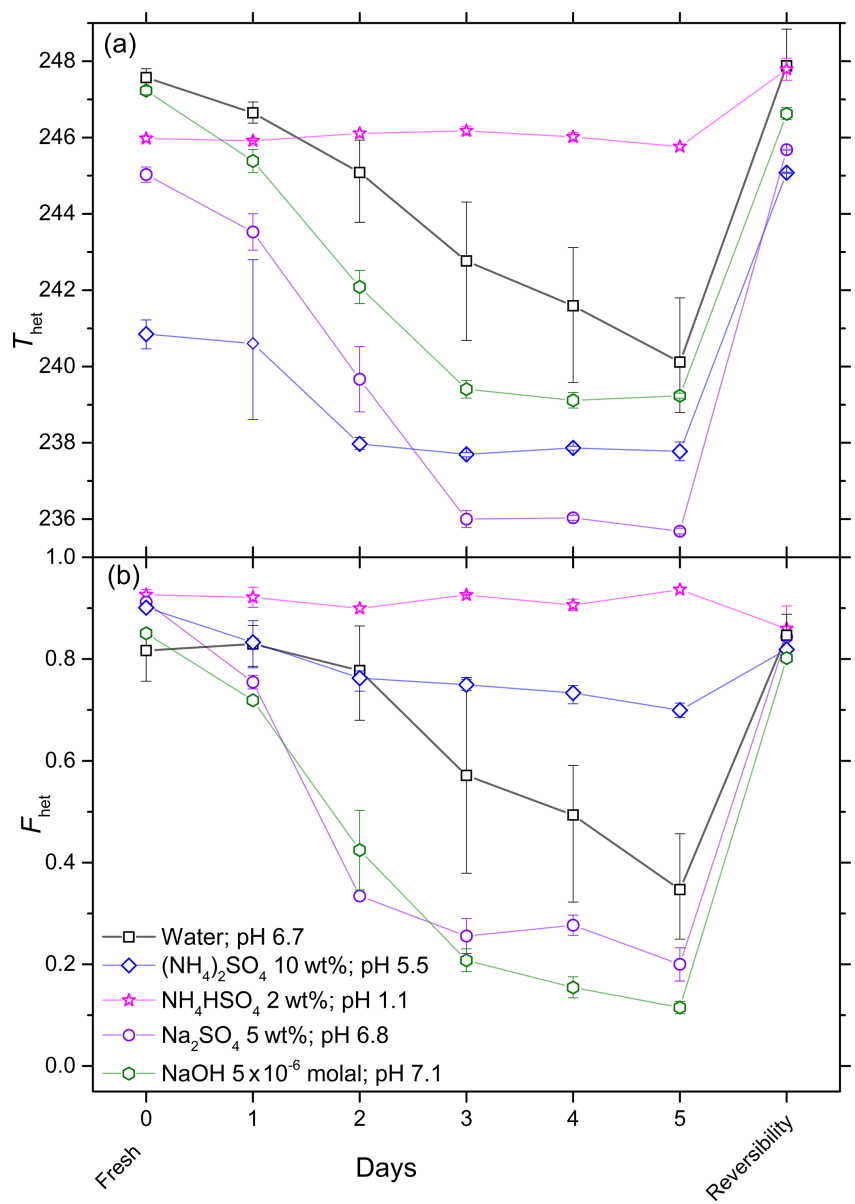

Figure 5. Development of $T_{\text {het }}$ (a) and $F_{\text {het }}$ (b) for $5 \mathrm{wt} \%$ SA quartz suspended in water, $\left(\mathrm{NH}_{4}\right)_{2} \mathrm{SO}_{4}$ solution (10 wt \%), $\mathrm{NH}_{4} \mathrm{HSO}_{4}$ solution (2 wt \%), $\mathrm{Na}_{2} \mathrm{SO}_{4}$ solution $(5 \mathrm{wt} \%)$ and $\mathrm{NaOH}$ solution $\left(5 \times 10^{-6}\right.$ molal $)$ over a period of 5 days. All suspensions were prepared and aged in borosilicate glass vials. After 5 days of aging the reversibility was tested: the suspensions were centrifuged, the supernatant decanted, the aged particles washed several times with pure water, resuspended in pure water, and subjected to an emulsion freezing experiment. All data points are means of at least two separately aged suspensions. The error bars show representative experimental variations ( $\min$ to $\max$ ).

turer) corroborate an increased IN efficiency in terms of $T_{\text {het }}$ and $F_{\text {het }}$ for the additionally milled sample as shown in Fig. 1 and Table 1. Moreover, milling of the amorphous silica sample from Alfa Aesar also had a very positive effect on its IN activity.

\subsection{Heterogeneous IN of quartz in aqueous solutions}

The water-activity-based description predicts heterogeneous IN temperatures $\left(T_{\text {het }}^{\Delta a_{\mathrm{w}}^{\text {het }}}\left(a_{\mathrm{w}}\right)\right)$ as a function of $a_{\mathrm{w}}$ by shifting the ice melting curve by a constant offset in $a_{\mathrm{w}}$. It is expected to be valid in the absence of specific interactions between the solute and the ice-nucleating surface so that the only effect of

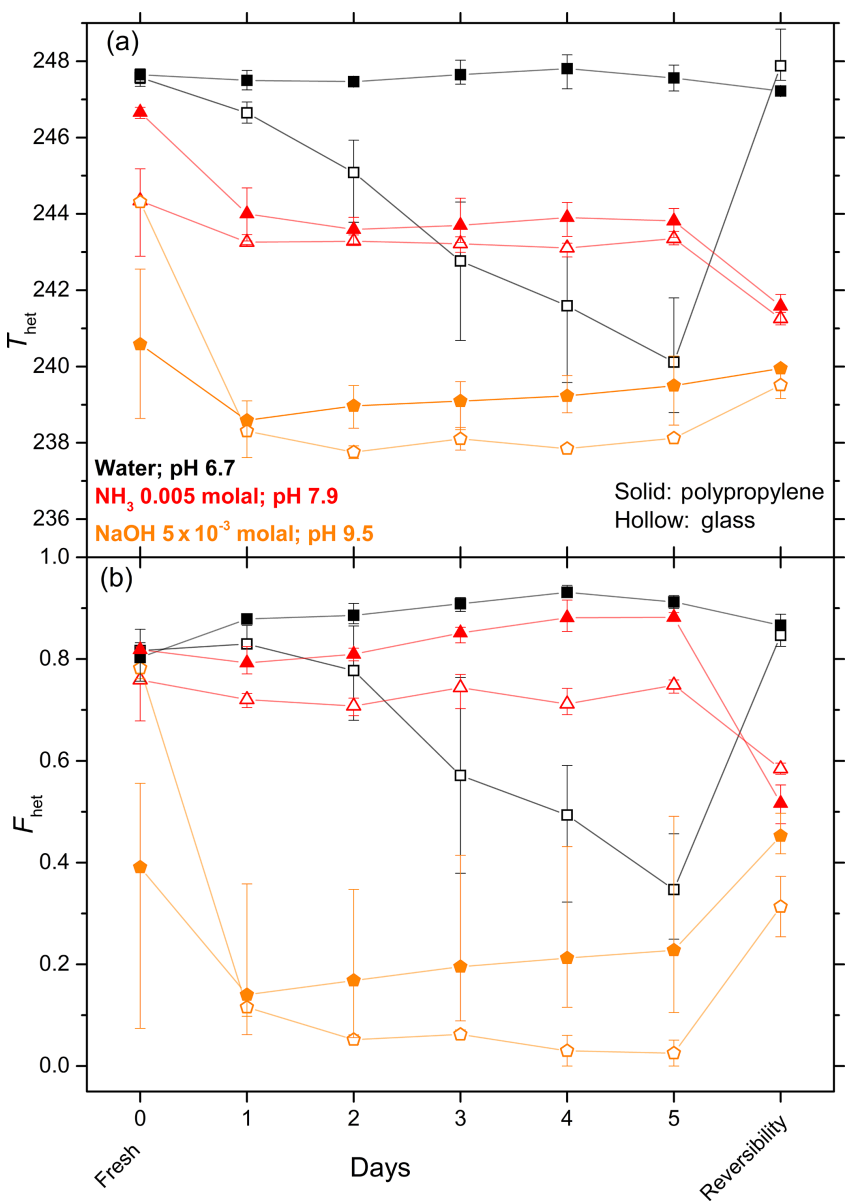

Figure 6. Same as Fig. 5, except that it shows a comparison of IN efficiency of SA quartz ( $5 \mathrm{wt} \%$ ) suspended in water/aqueous solutions $\left(\mathrm{NH}_{3} 0.005\right.$ molal with $\mathrm{pH} 7.9$ and $\mathrm{NaOH} 5 \times 10^{-3}$ molal with $\mathrm{pH}$ 9.5) prepared in borosilicate glass vials (open symbols) and polypropylene Falcon tubes (solid symbols). After 5 days aging the reversibility was tested as explained in Fig. 5.

the solute is a freezing point depression. Such a description has been suggested by several studies in the recent past $(\mathrm{Zu}-$ beri et al., 2002; Archuleta et al., 2005; Cantrell and Robinson, 2006; Zobrist et al., 2006, 2008; Alpert et al., 2011a, b; Knopf and Forrester, 2011; Rigg et al., 2013).

In Part 1 of this series, we showed that heterogeneous freezing onsets of microcline exhibit strong deviations from the water-activity-based description. Higher $T_{\text {het }}$ compared to predicted values were observed for microcline suspended in very dilute $\mathrm{NH}_{3} / \mathrm{NH}_{4}^{+}$-containing solutions, while a substantial decrease in IN efficiency was observed in more concentrated solutions of inorganic salts including $\mathrm{NH}_{4}^{+}$-containing salts and $\mathrm{NH}_{3}$ (Kumar et al., 2018a). In Part 3 (Kumar et al., 2019) we extended this investigation to other aluminosilicates and found that an increase in $T_{\text {het }}$ in the presence of $\mathrm{NH}_{4}^{+}$-containing solutes is a general feature of feldspars, clay minerals and micas, while the decrease in IN efficiency 
at higher solute concentration is a more specific characteristic of K-feldspars and most pronounced for microcline. Conversely, SA quartz follows quite well the water-activitybased prediction (black line in Fig. 2a) of $T_{\text {het }}$ in the case of $\mathrm{pH}$ neutral suspensions. $F_{\text {het }}$ shows an initial increase in very dilute solutions of $\left(\mathrm{NH}_{4}\right)_{2} \mathrm{SO}_{4}, \mathrm{NH}_{4} \mathrm{HSO}_{4}$ and $\mathrm{Na}_{2} \mathrm{SO}_{4}$, which is preserved to higher concentrations (Fig. 2b). Whale et al. (2018) compared the IN activity of quartz in dilute $\mathrm{NaCl}$ and $\left(\mathrm{NH}_{4}\right)_{2} \mathrm{SO}_{4}$ with the one in pure water and observed no change of the freezing onset temperatures in the presence of the solutes, but an increase in the density of IN active sites towards lower temperatures in a dilute $\left(\mathrm{NH}_{4}\right)_{2} \mathrm{SO}_{4}$ solution and a decrease in a dilute $\mathrm{NaCl}$ solution.

Opposite to the effect of $\mathrm{NH}_{3}$ on aluminosilicates, there is a decrease in $T_{\text {het }}$ for SA quartz in $\mathrm{NH}_{3}$ solutions. In the past, several infrared spectroscopy studies have investigated the adsorption of $\mathrm{NH}_{3}$ molecules on various types of mineral oxides (Mapes and Eischens, 1954; Eischens and Pliskin, 1958; Peri and Hannan, 1960). The quartz surface provides several centers for interaction with $\mathrm{NH}_{3}$ molecules viz. (i) hydrogen bonding via one of its hydrogen atoms with a surface oxygen atom of a silanol group; (ii) hydrogen bonding via its nitrogen atom with the hydrogen of a surface hydroxyl group and (iii) coordination to an electron-deficient $\mathrm{Si}$ (Lewis acid site) (Folman, 1961; Cant and Little, 1965; Blomfield and Little, 1973; Tsyganenko et al., 1975; Morrow and Cody, 1976; Morrow et al., 1976; Fubini et al., 1992; Li and Nelson, 1996; Wright and Walsh, 2012). In addition to reversible coordination to silanols via hydrogen bonding, $\mathrm{NH}_{3}$ may interact irreversibly with strained siloxane bridges by disrupting them into $\mathrm{Si}-\mathrm{NH}_{2}$ and $\mathrm{Si}-\mathrm{OH}$ groups (Folman, 1961; Peri, 1966), although, water displays more affinity than $\mathrm{NH}_{3}$ for this reaction (Blomfield and Little, 1973; Morrow and Cody, 1976; Fubini et al., 1992). Figure 2 shows that the sum of these interactions seems to affect the IN activity of the quartz surface by decreasing $T_{\text {het }}$ but slightly increasing $F_{\text {het }}$ at higher $\mathrm{NH}_{3}$ concentrations. Using sum frequency generation spectroscopy, Wei et al. (2002) have found an enhancement of the hydrogen bonded $\mathrm{OH}$ peak in the presence of ammonia. They proposed that $\mathrm{NH}_{3}$ molecules may form strong hydrogen bonds with the silanol groups on the silica surface with the nitrogen atoms facing silica, resulting in an excessive number of protons (NH bonds) pointing into ice. However, these interactions of $\mathrm{NH}_{3}$ with the quartz surface do not seem to lead to an enhanced IN activity compared with the pure water case.

Wright and Walsh (2012) found no strong and stable hydrogen bonding of $\mathrm{NH}_{4}^{+}$to hydroxylated quartz in their first principles molecular dynamics simulations. The slight decrease in $T_{\text {het }}$ in the presence of $\left(\mathrm{NH}_{4}\right)_{2} \mathrm{SO}_{4}$ together with the slight increase in $F_{\text {het }}$ indicate that the presence of ammonium in the solution influences the IN activity, although only slightly.

The decrease in IN activity in $\mathrm{NaOH}$-containing suspensions as shown in Fig. 2 can be ascribed to the detrimen- tal effect of alkaline conditions (pH between 7.1 and 13.6) on the stability of the quartz surface (House and Orr, 1992; Crundwell, 2017) and is discussed in the next section.

\subsection{Aging effect and reversibility of surface modifications}

The aging experiments performed with quartz suspended in different solutions point to surface processes that influence the IN activity of quartz over time. The IN activity was maintained over the whole aging period (5 days) in the case of $\mathrm{NH}_{4} \mathrm{HSO}_{4}$ (2 wt \%, $\left.\mathrm{pH} 1.1\right)$. In contrast, in pure water, $\left(\mathrm{NH}_{4}\right)_{2} \mathrm{SO}_{4}(10 \mathrm{wt} \%, \mathrm{pH} 5.5), \mathrm{Na}_{2} \mathrm{SO}_{4}(5 \mathrm{wt} \%, \mathrm{pH} 6.8)$ and very dilute $\mathrm{NaOH}\left(5 \times 10^{-6}\right.$ molal; pH7.1), the IN activity decreased over time but was completely or almost completely restored after washing in water (Fig. 5). In more alkaline solutions, namely $\mathrm{NH}_{3}(0.005$ molal, $\mathrm{pH} 7.9)$ and more concentrated $\mathrm{NaOH}\left(5 \times 10^{-3}\right.$ molal, $\left.\mathrm{pH} 9.5\right)$, IN activity was permanently lost (Fig. 6). Also, when quartz was aged for 7 months in pure water in glass vials, the IN activity was almost completely destroyed (Fig. 7). In contrast, the IN efficiency in pure water was barely affected during aging in polypropylene tubes over 5 days. Even after aging for 7 months the quartz sample proved to remain IN active. Yet, it showed a permanent decrease in IN efficiency $\left(T_{\text {het }}=243.4 \pm 1.6 \mathrm{~K}, F_{\text {het }}=0.59 \pm 0.1\right)$ that did not recover after washing in water, rather there was a slight further decrease to $T_{\text {het }}=242.4 \pm 2.5 \mathrm{~K}$ and $F_{\text {het }}=0.40 \pm 0.1$.

A decrease in IN activity due to aging for $72 \mathrm{~h}$ in glass vials in pure water was also observed for Kaufmann quartz (Fig. A2) and TU Vienna quartz (Fig. A3). In the following, we relate the results of the aging and reversibility experiments to surface processes occurring in the different solutions.

\subsubsection{Dissolution and growth of quartz in pure water}

Several studies have discussed the effect of solution $\mathrm{pH}$ on dissolution rates of quartz (Henderson et al., 1970; Kline and Fogler, 1981; Schwartzentruber et al., 1987; Bennett et al., 1988; Knauss and Wolery, 1988). The dissolution rate of quartz at $25^{\circ} \mathrm{C}$ is $10^{-13}$ to $10^{-12}$ moles $\mathrm{Si} \mathrm{m} \mathrm{m}^{-2} \mathrm{~s}^{-1}$ at low to neutral $\mathrm{pH}(0.5-7)$ and increases roughly linearly with increasing $\mathrm{pH}$, reaching a value of $10^{-10}$ moles $\mathrm{Si} \mathrm{m}^{-2} \mathrm{~s}^{-1}$ at pH 12 (House and Orr, 1992; Crundwell, 2017). The dissolution of quartz is considered to occur on deprotonated silanols, i.e., $\mathrm{Si}-\mathrm{O}^{-}$(Brady and Walther, 1989, 1990). Deprotonation of the silanol weakens the remaining siloxane bridges, facilitating the attack by water molecules and ultimately releasing the $\mathrm{Si}$ in the form of silicic acid $\left(\mathrm{H}_{4} \mathrm{SiO}_{4}\right)$. Moreover, dissolution of quartz increases with increasing ionic strength of the solution, i.e., increasing salt concentration (Brady and Walther, 1990). At low $\mathrm{pH}$, different dissolution mechanisms may be involved, such as $\mathrm{H}_{2} \mathrm{O}$ hydrolysis of $\mathrm{Si}$ centers or adsorption of $\mathrm{H}^{+}$onto siloxane bridging oxygen (Xiao and 


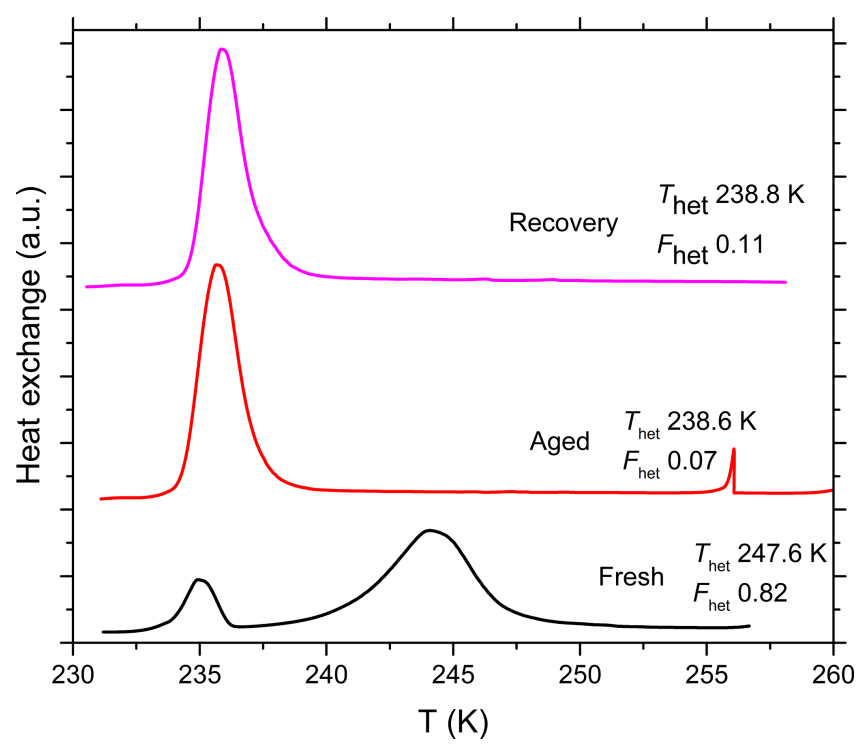

Figure 7. DSC thermograms of SA quartz (5 wt \%) suspended in pure water in borosilicate glass vials and measured right after preparation (marked as "Fresh"). The same suspension was aged for 7 months and remeasured (marked as "Aged"). The aged suspension was centrifuged, the supernatant decanted, the aged particles washed several times with pure water, resuspended in pure water and measured again to examine the recovery of IN efficiency after aging (marked as "Recovery"). This procedure was done with three different suspensions and their mean $T_{\text {het }}$ and $F_{\text {het }}$ are reported next to each curve. All curves are normalized such that the areas under the heterogeneous and homogeneous freezing curves sum up to the same value.

Lasaga, 1994; Criscenti et al., 2006; Bickmore et al., 2008), but with a low efficiency.

The SA quartz as provided by the manufacturer contains a minor share of amorphous material $(4.5 \pm 0.5 \%)$, produced most likely by grinding (Fubini et al., 1989). Because of the higher dissolution rate of amorphous silica compared to quartz, silicic acid should be released at a higher rate from the amorphous material (Crundwell, 2017). Since also the solubility of amorphous silica is higher $(\sim 50 \mathrm{ppm}$ $\mathrm{Si}$ at $\left.25^{\circ} \mathrm{C}\right)$ than the one of quartz $\left(1-3 \mathrm{ppm} \mathrm{Si}\right.$ at $\left.25^{\circ} \mathrm{C}\right)$ (Walther and Helgeson, 1977), quartz should grow at the expense of amorphous silica over time when kept together in a closed vessel. After aging for $72 \mathrm{~h}$ in water (in a glass vessel), the amorphous fraction of the SA quartz sample indeed showed a slight decrease, but still within experimental error ( $4.0 \pm 0.5 \%$ for the aged sample compared with $4.2 \pm 0.5 \%$ for the sample exposed to water for $\sim 15 \mathrm{~min}$ ). This is in agreement with the fact that the conversion of amorphous silica to quartz is a slow process.

ICP-MS measurements (see Tables S1 and S2 in the Supplement) performed with the supernatant of a $0.5 \mathrm{wt} \% \mathrm{SA}$ quartz suspension that was aged in pure water for $72 \mathrm{~h}$ in a borosilicate glass vial shows a concentration of $11.9 \mathrm{ppm} \mathrm{Si}$, which is well above the solubility of quartz in pure water and well below that of amorphous silica. The $\mathrm{Si}$ concentration after aging a $0.7 \mathrm{wt} \%$ SA quartz suspension for $72 \mathrm{~h}$ in polypropylene Falcon tubes reaches only $4.9 \mathrm{ppm}$, indicating that a considerable fraction of the dissolved $\mathrm{Si}$ stems from the glass vial in the aged sample. Indeed, the glass vial continuously leaches Si to the water (Bunker, 1994). The ICPMS measurement of pure water, which was in contact with the glass vial for less than an hour, shows a concentration of only $0.2 \mathrm{ppm}$ Si but reached $4.5 \mathrm{ppm} \mathrm{Si}$ after $72 \mathrm{~h}$. This slower increase in Si concentration compared to the immediate increase in the case of the quartz samples (1.3-6 ppm after less than an hour in water) is simply due to the smaller surface area of the glass vial ( $\sim 2$ orders of magnitude) exposed to water compared with the one of the SA quartz sample. In addition, also the release of Si from the quartz sample is expected to stem mostly from its amorphous share. Subsequently, silicic acid in water may form dimers, trimers and cyclic species due to autopolycondensation that sets in when the silicic acid concentration approaches the solubility limit (Perry, 1989, 2003; Belton et al., 2012). These oligomerization reactions are reversible (Tamahrajah and Brehm, 2016). We assume that at high Si concentration, silicic acid and its oligomers adsorb on the quartz surface, covering large parts of the crystalline surface or at least a relevant fraction of the IN active sites, thus hampering the IN activity of the quartz samples aged in glass vials. Indeed, monolayer adsorption of silicic acid on quartz has been observed under conditions supersaturated with respect to crystalline quartz (Berger et al., 1994). Since leaching of $\mathrm{Si}$ from the glass vials is slow owing to the comparatively small exposed surface area, the freshly prepared suspensions of SA quartz in glass vials are not affected by an adsorbed layer, which is in accordance with our experiments (see Figs. 5 and 6). The Si concentration in the polypropylene tubes remains too low to give rise to an adsorbed layer on the quartz surface even during aging and the IN activity is not hampered (see Fig. 6).

With time, the Si supersaturation with respect to quartz should lead to crystalline quartz growth. We assume that covalent bonds form between the adsorbed siliceous layer and the quartz surface leading to a grown, intact quartz surface with few defects. Since the emulsion freezing experiments of the SA quartz sample aged for 7 months in a glass vial show hardly any IN activity even after washing with pure water (see Fig. 7), we conclude that slowly grown quartz surfaces are indeed not amorphous, but have a regular crystalline structure. These are barely IN active and only milling provides the quartz surface with IN active sites.

\subsubsection{Dissolution and growth of quartz in solutions}

Under alkaline conditions both quartz dissolution and growth rates are increased. Indeed, centimeter-sized synthetic quartz crystals are grown from amorphous silica on seed crystals at high temperatures and pressures in highly alkaline con- 
ditions (Baughman, 1991). Such rigorous conditions are applied to accelerate the crystal growth. In the $5 \times 10^{-3}$ molal $\mathrm{NaOH}$ solution ( $\mathrm{pH} 9.5$ ), the solubility of amorphous silica and quartz are higher than at near-neutral conditions and dissolution and growth of quartz are enhanced. We therefore ascribe the strong and immediate loss of IN activity of SA quartz suspended in $5 \times 10^{-3}$ molal $\mathrm{NaOH}$ solution in polypropylene tubes to the dissolution of quartz, which levels off when the equilibrium condition is approached. Therefore, during the following days of aging, there is no further decrease in IN activity, rather $T_{\text {het }}$ and $F_{\text {het }}$ show a slight increase (but within the uncertainty limits). After washing with water, $F_{\text {het }}$ recovers to the initial value in $5 \times 10^{-3}$ molal $\mathrm{NaOH}$ but remains clearly below the value measured in pure water. When the experiment is carried out in glass vials (in $5 \times 10^{-3}$ molal $\mathrm{NaOH}$ ), the initial loss of IN activity is less pronounced, probably because the additional leaching of $\mathrm{Si}$ from the glass vial leads to a quick increase in the Si concentration above the saturation level with respect to quartz. As a consequence, the quartz sample is in growth conditions for most of the aging time and we ascribe the irreversible loss of IN activity to growth of intact quartz layers, which is faster than at neutral conditions because of the higher Si solubility under alkaline conditions.

Interestingly, during aging in $\mathrm{NH}_{3}$, the main decrease in $T_{\text {het }}$ is observed after one day while $F_{\text {het }}$ is preserved during the 5 days of aging but is reduced when $\mathrm{NH}_{3}$ is removed from the suspension. This implies that the presence of $\mathrm{NH}_{3}$ can temporarily stabilize the surface followed by a strong decrease when it is removed.

\subsection{Which factors determine the IN activity of quartz?}

\subsubsection{Crystallinity and substrate-ice lattice match}

Crystallinity and lattice match between substrate and ice are often considered to provide IN activity to the substrate. While ice and silica exhibit structural similarities in the form of tetrahedral building units, the most common hexagonal (Ih) and cubic (Ic) ice phases show structural analogies to the crystalline silica tridymite and cristobalite, respectively, but not to quartz (Tribello et al., 2010). Indeed, when the quartz surface is not produced by milling but by crystal growth, quartz is similarly inactive as amorphous silica. Conversely, when hardly active amorphous silica particles are milled, they become IN active. This suggests that the regular quartz surface is not able to template ice growth and that the crystallinity of quartz is not a prerequisite for its IN activity.

\subsubsection{Milling and radical site formation}

Micrometer- and nanometer-sized quartz particles are usually obtained by milling. Due to the covalent nature of the quartz crystal lattice, considerable force needs to be exerted to obtain small particles by milling. The shear and com- pression applied to quartz leads to a disturbed amorphous zone of $10-30 \mathrm{~nm}$ thickness with dangling $\mathrm{Si}-\mathrm{O}^{\bullet}$ and $\mathrm{Si}^{\bullet}$ radical sites that can be detected by electron paramagnetic resonance (EPR) (Fubini et al., 1987, 1989; Makoto and Motoji, 1996). When quartz is ground in the normal atmosphere, the resulting $\mathrm{Si}-\mathrm{O}^{\bullet}$ and $\mathrm{Si}^{\bullet}$ radical sites react with atmospheric species (Fubini et al., 1987). Reactions with $\mathrm{O}_{2}$ lead to the formation of $\mathrm{Si}-\mathrm{O}-\mathrm{O}^{\circ}, \mathrm{Si}-\mathrm{O}-\mathrm{O}-\mathrm{Si}$ or $\mathrm{Si}-\mathrm{O}-\mathrm{O}-\mathrm{O}-\mathrm{Si}$, which react in the presence of water (vapor) to $\mathrm{Si}-\mathrm{OH}$ and $\mathrm{Si}-\mathrm{O}-\mathrm{OH}$. In addition, hydroxylation of strained $\mathrm{Si}-\mathrm{O}-\mathrm{Si}$ groups (Fubini et al., 1987) results in silanol groups $(\equiv \mathrm{Si}-\mathrm{OH})$ rendering the surface highly hydroxylated. Depending on their arrangement on the silica surface, silanols are isolated $\left((\mathrm{Si}-\mathrm{O})_{3} \mathrm{Si}-\mathrm{OH}\right)$, germinal $\left(=\mathrm{Si}(\mathrm{OH})_{2}\right)$ or vicinal $(=\mathrm{Si}(\mathrm{OH})-\mathrm{O}-\mathrm{Si}(\mathrm{OH})=)$. The relatively non-polar siloxane bridges $(\equiv \mathrm{Si}-\mathrm{O}-\mathrm{Si} \equiv)$ may be strained and easily hydrolyzed to silanols or regular and hardly reactive (Morrow and Cody, 1976; Morrow et al., 1976; Brinker et al., 1986, 1988; Zhdanov et al., 1987; Bolis et al., 1991; Fubini et al., 1992).

The IN activity observed for milled Alfa Aesar silica suggests that shear and compression applied to amorphous silica leads to similar radical sites as in the case of quartz, providing amorphous silica with IN activity. XRD analysis of Kaufmann quartz and SA quartz support the presence of amorphous shares of about $6.4 \mathrm{wt} \%$ and $4.5 \mathrm{wt} \%$ in the samples, respectively. A part of this amorphous material may agglomerate as separate particles or in specific regions of the quartz surface. In the Supplement, we show a collection of representative SEM images of SA and Kaufmann quartz that show agglomerates on top of the quartz surface, which might be amorphous. Nevertheless, an amorphous surface layer on top of the quartz particles cannot be excluded. Because of the strong correlation of milling and IN activity of silica surfaces, we propose that the surface functionalization of silica particles arising from breaking covalent $\mathrm{Si}-\mathrm{O}$ bonds during milling gives rise to the IN activity of these materials.

Interestingly, the conjecture that surface functionalization resulting from the cleavage of covalent $\mathrm{Si}-\mathrm{O}$ bonds rather than the ordered crystalline structure determines the surface properties of silica is confirmed by findings from a completely different research field. Ground quartz particles may induce silicosis, lung cancer and autoimmune diseases (Donaldson and Borm, 1998; Fubini, 1998). This pathogenicity is totally absent in chemically prepared amorphous silica or synthesized (grown) quartz particles. Since exposure to ground amorphous (vitreous) silica also leads to adverse health effects (Turci et al., 2016), the pathogenicity is considered to arise rather from the mechanical cleavage of the covalent $\mathrm{Si}-\mathrm{O}$ bonds than from the crystallinity (Fubini et al., 1987, 1989; Turci et al., 2016). 


\subsubsection{Surface $\mathrm{OH}$ groups}

While siloxanes and silanols dominate the surfaces of amorphous silica and quartz, the relative surface densities of these groups show large variation. Fully hydroxylated quartz surfaces may carry up to $9.5 \mathrm{OH} \mathrm{nm}^{-2}$ on the (001) surface and still $5.8 \mathrm{OH} \mathrm{nm}^{-2}$ on the (011) and (101) surfaces (Musso et al., 2009). On the other hand, fully hydroxylated amorphous silica surfaces carry typically only $4.6-4.9 \mathrm{OH} \mathrm{nm}^{-2}$. Highly hydroxylated surfaces are dominated by vicinal and geminal silanols, with few isolated silanol and siloxane groups (Zhuravlev, 2000; Muster et al., 2001). Due to their more ordered structure, silanols on quartz surfaces tend to form networks of chains of hydrogen bonds, whereas amorphous silica surfaces rather exhibit patches of hydrogen bonded silanols, even if their average silanol densities are the same (Musso et al., 2011).

When quartz and silica samples are heated, surface hydroxylation decreases due to the replacement of silanols by siloxanes. The more severe the heating conditions (temperature, vacuum, duration), the more dehydroxylized the surface becomes. Heating (calcination) to $670 \mathrm{~K}$ in vacuum removes all vicinal silanols of amorphous silica while isolated silanols are still present but become continuously scarcer by further heating (Zhuravlev, 2000). Quartz, on the other hand, is less easily dehydroxylated (Bolis et al., 1985). Dehydroxylated surfaces slowly rehydroxylate when they are exposed to humidity or in contact with liquid water. The Stöber particles shown in Fig. 1 have been heated to $823 \mathrm{~K}$, which strongly decreased the number of vicinal silanols and subsequently hydrolyzed so that vicinal silanols should be restored to a full hydroxylation level of amorphous silica (i.e., 4.6-4.9 $\left.\mathrm{OH} \mathrm{nm}^{-2}\right)$.

Musso et al. (2012) showed in an ab initio molecular dynamics study that the silanol surface of quartz (100) induces an ice-like structure of water in the proximity of the surface, which is more pronounced when the silanol density at the surface is higher. While water molecules spontaneously form hydrogen bonds to isolated silanols, hydrogen bonding to vicinal silanols involves breaking the surface hydrogen bond network between them. This is an activated process with an activation energy that increases with increasing length of the interconnected silanol chains (Musso et al., 2011). Water was only able to disrupt the weak internal hydrogen bonds between surface silanols with $\mathrm{H} \cdots \mathrm{O}>2 \AA$ but not the stronger ones with $\mathrm{H} \cdots \mathrm{O}<2 \AA$ (Musso et al., 2012). Milling decreases the long-range order of silanols and generates hydrophilic and hydrophobic patches (Turci et al., 2016). The generated defects may disrupt the chain of interconnected silanols and free them to participate in hydrogen bonding with water molecules. This could explain the enhanced IN activity of freshly milled quartz as well as freshly milled amorphous silica.

Recently, it had been suggested that the $\mathrm{OH}$ density and the substrate-water interaction strength are useful descrip- tors of a material's IN ability (Pedevilla et al., 2017). The example of quartz shows that $\mathrm{OH}$ density alone is indeed insufficient as a predictor for IN ability when strong hydrogen bonding amongst surface $\mathrm{OH}$ groups prevail over substratewater interactions.

\subsubsection{Protonation or deprotonation of surface $\mathrm{OH}$ groups and surface charge}

Depending on the solution $\mathrm{pH}$, the silanol groups protonate or deprotonate, thus changing the surface charge. The quartz surface is at the point of zero charge (PZC) around $\mathrm{pH} 2$ and becomes more negative with increasing $\mathrm{pH}$ (Vidyadhar and Hanumantha Rao, 2007; Turci et al., 2016). At PZC, Si-OH groups prevail, and the number of $\mathrm{Si}-\mathrm{OH}_{2}^{+}$equals the number of $\mathrm{Si}-\mathrm{O}^{-}$groups. The ordering of water at the quartz surface was shown to be $\mathrm{pH}$ dependent. At $\mathrm{pH} 1.5$ when the surface is slightly positively charged and at $\mathrm{pH} 12.3$ when the surface is negatively charged, water molecules are ordered but the orientation is reversed from low to high $\mathrm{pH}$. At intermediate $\mathrm{pH}$, there is more disorder (Du et al., 1994; Richmond, 2002). Zeta potential measurements show that the milled quartz surface is slightly less negatively charged than grown quartz surfaces at neutral conditions. However, milling increases the heterogeneity of the silanols and creates more acidic sites as indicated by a shallower increase in surface charge with decreasing pH (Turci et al., 2016).

Besides surface functionalization, surface charge is a factor that has been shown to influence IN activity (Marcolli et al., 2016; Abdelmonem et al., 2017; Kumar et al., 2019). Abdelmonem et al. (2017) found that the freezing temperature of water at the $\alpha-\mathrm{Al}_{2} \mathrm{O}_{3}$ (0001) surface is highest when the surface is close to the PZC and the water molecules at the surface are least ordered. In contrast, Kumar et al. (2019) shows that hydroxylated surfaces that had PZCs at low $\mathrm{pH}$ such as feldspars showed higher IN activity in pure water than surfaces with PZC shifted to neutral or alkaline conditions.

\subsubsection{Impurities on mineral surfaces}

Often IN activity of mineral surfaces has been related to the presence of impurities introducing special sites on the mineral surface (O'Sullivan et al., 2014; Zolles et al., 2015). Milling can accumulate impurities from within the crystal lattice (DeMott et al., 2003b; Boose et al., 2016a) on the surface because regions rich in impurities provide preferred cleavage planes (Whale et al., 2017). If such impurities are surface active and remain adsorbed on quartz when the particles are immersed in water, they can either block active sites and decrease the IN activity or generate new active sites. Milling of quartz leads to high energy surface sites that may attract semivolatile impurities to reduce the surface energy. Indeed, organic semivolatile material has been considered to provide IN activity to mineral surfaces (O'Sullivan et al., 2014; Tobo et al., 2014). 
We therefore determined the presence of semivolatile material on the Kaufmann and SA quartz samples by performing TGA. Since only a mere $0.17 \%$ and $0.30 \%$ loss in weight was observed in TGA up to $350^{\circ} \mathrm{C}$ (the Supplement) in Kaufmann and SA quartz samples, respectively, the influence of semivolatile material on the IN efficiency is unlikely. To investigate the possibility that nonvolatile but water-soluble impurities provide IN activity to the quartz surface, we performed ICP-MS tests on the supernatant liquid of quartz suspensions $(0.7 \mathrm{wt} \%)$ freshly prepared in water in polypropylene Falcon tubes. Si had a contribution of more than $80 \%$ of the leached elements in both quartz cases (see the Supplement; Table S2 for details). The most abundant impurities were $\mathrm{Na}, \mathrm{Al}, \mathrm{K}, \mathrm{Ca}, \mathrm{Ba}, \mathrm{Fe}$ and $\mathrm{Co}$ with a combined contribution of only $12 \%$ and $18 \%$ to the total leached elements for SA and Kaufmann quartz, respectively. Depletion of these elements from the quartz surface cannot explain the loss of IN activity during aging because the IN activity can be restored by washing the quartz particles with pure water. We therefore regard the abundance of $\mathrm{Si}-\mathrm{OH}$ groups and their arrangement on the quartz surface relevant for IN activity in emulsion freezing experiments rather than the presence of foreign components. However, we do not exclude that IN activity observed at higher temperatures in bulk freezing experiments might be due to impurities (O'Sullivan et al., 2014). Indeed, the notion that different types of sites are relevant at higher than at lower freezing temperatures is corroborated by Kaufmann et al. (2016) who showed in their Fig. 6 that only a weak correlation exists between freezing temperatures of bulk and emulsion freezing experiments of different mineral dusts.

From the above discussion, we conclude that milling is a requirement for quartz to be IN active and that IN occurs on specific active sites introduced by milling, which are more reactive than the grown quartz surface. Despite the high degree of hydroxylation, the regular quartz surface does not give rise to IN activity, likely because most silanols are tightly interconnected by hydrogen bonds in a network that is too strong to be disrupted by water molecules.

\section{Atmospheric implications}

Arid and semiarid regions are the main sources of mineral dust (e.g., Saharan and Gobi deserts) (Laurent et al., 2006, 2008), which can have atmospheric lifetimes of several days (Huneeus et al., 2011). The dust, while being transported, can interact with a variety of trace gases (Usher et al., 2003; Kolb et al., 2010), which can lead to changes in the surface physicochemical properties. Not only ground-collected and/or near-source but also transported dusts have been reported to be rich in quartz (Avila et al., 1997; Alastuey et al., 2005; Field et al., 2006; Boose et al., 2016b; Kaufmann et al., 2016). Quartz can be found in high proportions in atmospherically transported Saharan dust samples (Avila et al., 1997;
Caquineau et al., 1998; Caquineau et al., 2002; Alastuey et al., 2005; Kandler et al., 2009). However, barely any information is available about the alteration of the IN efficiency of quartz due to cloud processing and atmospheric chemical species.

This work and Zolles et al. (2015) have shown a large variability in the IN activity of quartz particles. Milled quartz samples showed high IN activity, while quartz layers grown over 7 months were almost IN inactive. This is in agreement with the low IN activity of a natural quartz sample investigated by Zolles et al. (2015) with freezing onset $<238 \mathrm{~K}$. The question therefore arises whether naturally eroded quartz particles from atmospherically relevant dust source regions would have significant IN activity.

Kaufmann et al. (2016) performed emulsion freezing experiments with natural dust samples ground-collected in different deserts worldwide and correlated the IN activity with the mineralogical composition. A sample collected in Oman showed low IN activity despite its considerable quartz content. Conversely, heterogeneous freezing on quartz surfaces may indeed account for the freezing signal observed for higher suspension concentrations of desert dust samples collected in Israel. For the Antarctica sample with $24 \%$ quartz content, Kaufmann et al. (2016) performed emulsion freezing experiments using the sieved fraction and after the sieved fraction was milled. Indeed, the heterogeneously frozen fraction increased due to milling.

Boose et al. (2016b) found a positive correlation between the quartz content and the freezing between 238 and $245 \mathrm{~K}$ of dust particles sampled from deserts worldwide. However, this positive correlation strongly relies on two milled samples with the highest quartz content $(\sim 64 \%$ and $\sim 93 \%$ in samples from Australia and Morocco), which exhibit the highest IN activity. In addition, two more samples were sieved and milled as well. In the case of the Atacama sample, with quartz content of $\sim 17 \%$ after sieving and $10 \%$ after milling, milling increased the IN activity. In the case of the Israel sample with quartz contents of $\sim 7 \%$ after sieving and $\sim 6 \%$ after milling, the IN activity was decreased after milling. This shows that the IN activity of collected mineral dusts depends in a complex way on the preprocessing of the samples. More samples need to be collected from desert regions and tested for IN activity without prior milling to assess the correlation between IN activity and quartz content.

\section{Conclusions and outlook}

The analysis of emulsion freezing experiments of quartz and amorphous silica particles allowed us to attribute the IN activity of quartz surfaces to specific surface properties.

Surface hydroxylation seems to be a necessary but not sufficient condition for the IN activity of quartz and amorphous silica. Silanols may form hydrogen bonds with water molecules that are able to direct them into an ice-like 
arrangement. However, as it seems, most of the silanols on synthesized amorphous silica and grown quartz surfaces are engaged in hydrogen bonds amongst each other with an insufficient number of $\mathrm{OH}$ groups left for hydrogen bonding to water molecules.

Milling leads to the cleavage of $\mathrm{Si}-\mathrm{O}-\mathrm{Si}$ bridges resulting in $\mathrm{Si}-\mathrm{O}^{*}$ and $\mathrm{Si}^{\bullet}$ radical sites that react in the presence of water vapor to finally form $\mathrm{Si}-\mathrm{OH}$ and $\mathrm{Si}-\mathrm{O}-\mathrm{OH}$ on the surface. Defects seem to disrupt the interconnected chains of silanols on the surfaces of milled silica particles, thus increasing the number of silanols that are available for hydrogen bonding to water molecules. These defects indeed seem to be a prerequisite for the IN activity of amorphous silica and quartz.

Usually, amorphous silica particles are synthesized and quartz particles are milled, therefore the crystalline surface of quartz could be expected to match ice. Our emulsion freezing experiments with milled amorphous silica and grown quartz surfaces show that crystallinity is not relevant for the IN activity of silica. Rather a highly defective surface is required.

Both barely IN active grown quartz and highly IN active milled quartz particles carry a negative surface charge at neutral $\mathrm{pH}$ conditions. This indicates that surface charge alone is unreliable as a predictor for IN activity.

The onset freezing temperatures of quartz suspensions freshly prepared in neutral and acidic salt solutions containing $\left(\mathrm{NH}_{4}\right)_{2} \mathrm{SO}_{4}, \mathrm{NH}_{4} \mathrm{HSO}_{4}$ and $\mathrm{Na}_{2} \mathrm{SO}_{4}$ follow approximately the prediction of the water-activity-based description, while the heterogeneously frozen fraction increases slightly in the presence of these salts.

The IN activity of quartz is decreased in alkaline solutions. The interaction with $\mathrm{NH}_{3}$ suppresses the IN activity of the quartz surfaces. This is in contrast to the increased IN activity of gibbsite and aluminosilicates, i.e., feldspars, mica and kaolinite in the presence of $\mathrm{NH}_{3}$. We ascribe this decrease in IN activity to the increased dissolution of quartz under alkaline conditions. The defects that constitute the active sites seem to be more susceptible and therefore disappear first on a dissolving surface.
Suspending quartz particles over days in aqueous solutions at near-neutral $\mathrm{pH}$ conditions in a glass vial decreases the IN activity considerably. This decrease is reversible and the original IN activity in terms of $F_{\text {het }}$ is almost restored when the quartz particles are washed and resuspended in pure water. We assume that a part of the silicic acid leached from the glass vial forms an oligomerized silicic acid layer on top of the quartz surface and blocks the active sites. This layer dissolves when the particles are rinsed in pure water. The formation of this layer might be the first step to quartz growth.

The sensitivity of the IN activity of quartz surfaces to environmental conditions makes it difficult to come to general conclusions regarding the relevance of quartz particles for cloud glaciation. Dry erosion may fracture quartz particles and introduce active sites while wet erosion may destroy active sites. To assess the IN activity of airborne quartz particles, a correlation between the quartz content and the IN activity must be established for samples that did not undergo milling before they were tested for freezing.

Data availability. The data for freshly prepared quartz suspensions in water or aqueous solutions (Fig. 2) and aging tests (Figs. 5 and 6) presented in this publication are available at https://doi.org/10.3929/ethz-b-000286931 (Kumar et al., 2018b). 


\section{Appendix A: Ice nucleation efficiency of quartz from Kaufmann et al. (2016)}

A recent study from our group, Kaufmann et al. (2016), reported a very low IN efficiency of a quartz sample suspended in pure water using the same experimental equipment and procedure as in the present study. In emulsion freezing experiments, a heterogeneous freezing signal was observed up to $\sim 247 \mathrm{~K}$, yet with a very low IN active particle fraction (0.01). The quartz sample used in Kaufmann et al. (2016) was procured as a stone from the Institute of Geochemistry and Petrology of ETH Zurich and milled to a fine powder in a tungsten carbide ball mill. Approximately $0.4 \mathrm{wt} \%$ of tungsten carbide was introduced as an impurity in the quartz sample due to milling.

To explain the low IN efficiency of the Kaufmann quartz compared with SA quartz, we further characterized these two samples. Scanning electron microscopy (SEM) revealed the presence of giant particles (diameter $>20 \mu \mathrm{m}$ ) in the milled quartz sample from Kaufmann et al. (2016) as shown in Fig. A1, which were absent in SA quartz. SA quartz is dominated by particles ranging from 0.5 to $10 \mu \mathrm{m}$ as specified by Sigma-Aldrich. As a hard mineral, quartz is difficult to mill down to very fine particles without partial amorphization. The amorphous fraction of the Kaufmann quartz was estimated as $6.4 \pm 0.5 \%$ based on the background signal observed in the XRD diffractogram compared to $4.5 \pm 0.5 \%$ for the SA quartz.

The size distribution measurements performed with SMPS/APS in Kaufmann et al. (2016) did not capture the coarse particle fraction due to inefficient aerosolization of large particles in the fluidized bed. Even when aerosolized, coarse particles can sediment in the tubing and connections during transport to the SMPS/APS. When calculating the fraction of droplets filled with particles, the number of particles per sample mass was highly overestimated because the coarse particles did not appear in the size distribution determined by SMPS/APS. Therefore, in Kaufmann et al. (2016), the homogeneous freezing signal observed in the emulsion freezing experiments was wrongly assigned to droplets filled with IN inactive quartz particles although it was mostly due to empty droplets.

To remove the coarse particles present in the Kaufmann quartz, we suspended a concentrated suspension of this sample for an hour in pure water so that particles with average diameters $\geq 3 \mu \mathrm{m}$ should settle. After this time period, the supernatant was collected and immediately used for an emulsion freezing experiment in order to avoid any further aging of the particles. The concentration of the supernatant was determined as $\sim 8 \mathrm{wt} \%-9 \mathrm{wt} \%$ by evaporating the water and weighing the dried residual.

In addition, emulsion freezing experiments were carried out on the same supernatant suspension aged for 2, 24 and $72 \mathrm{~h}$. The DSC thermograms of these measurements are shown in Fig. A2. The heterogeneous freezing onset tem-
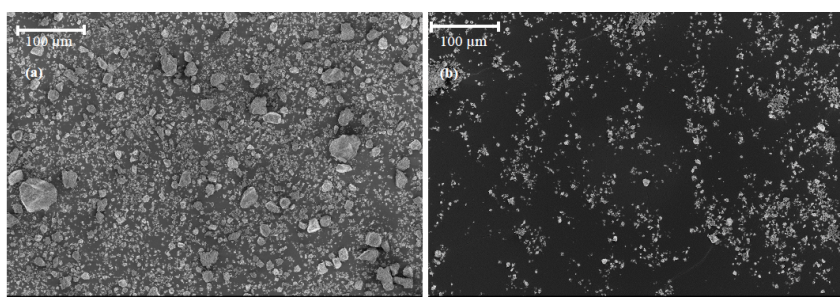

Figure A1. SEM images of two different quartz samples at 500× magnification. (a) Quartz sample from Kaufmann et al. (2016); (b) quartz sample (from Sigma-Aldrich) used in this study.



Figure A2. DSC thermograms of Kaufmann quartz taken from the supernatant of a suspension of the same quartz sample as used in Kaufmann et al. (2016) that had settled for $1 \mathrm{~h}$. Settling resulted in particle sizes with diameter $\leq 3 \mu \mathrm{m}$ and a suspension concentration of $8 \mathrm{wt} \%-9 \mathrm{wt} \%$ in the supernatant. In order to assess the effect of aging, emulsion freezing experiments were performed on the supernatant right after extraction as well as after 2,24 and $72 \mathrm{~h}$ after extraction and the corresponding $T_{\text {het }}$ and $F_{\text {het }}$ is reported next to each curve. All curves are normalized such that the areas under the heterogeneous and homogeneous freezing curves sum up to the same value. The ${ }^{*}$ symbol represents the onset temperatures of the two shoulders of the heterogeneous freezing signal and the corresponding $F_{\text {het }}$ is calculated based on the complete freezing signal.

perature of the fresh supernatant was $250.2 \mathrm{~K}$, which is even higher than the $T_{\text {het }}$ of SA quartz, while the heterogeneously frozen fraction is almost the same for these two quartz samples. This confirms that the low IN efficiency of quartz reported in Kaufmann et al. (2016) was biased by the presence of giant quartz particles not captured in the SMPS/APS measurements. 


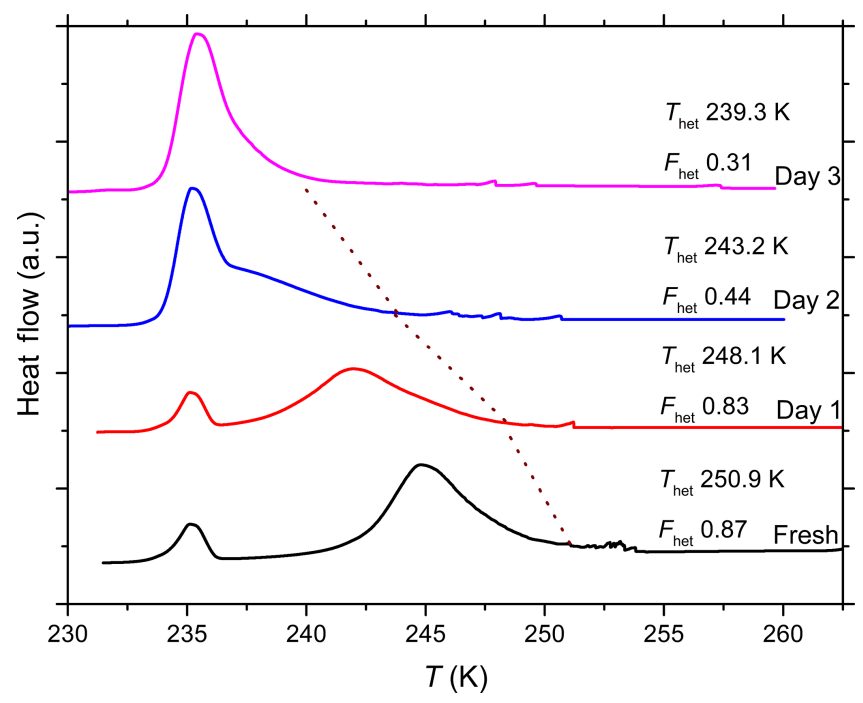

Figure A3. DSC thermograms of $5 \mathrm{wt} \%$ TU Vienna quartz suspension in pure water, obtained via emulsion freezing experiments performed on the day of suspension preparation (fresh) and the subsequent 3 days and the corresponding $T_{\text {het }}$ and $F_{\text {het }}$ are reported next to each curve. Suspensions were prepared in borosilicate glass vials. All curves are normalized such that the areas under the heterogeneous and homogeneous freezing curves sum up to the same value.

\section{Appendix B: Aging tests on TU Vienna quartz suspended in pure water}

Suspensions of TU Vienna quartz (5 wt \%) in pure water were prepared in glass vials and tested over a period of 3 days. Immersion freezing experiments were carried out with the DSC setup with emulsions prepared from at least two separate suspensions. Measurements were done on the day of preparation (fresh) and on the subsequent 3 days in order to assess the long-term effect of aging on the IN efficiency of the quartz sample. Figure A3 shows the DSC thermograms from freezing experiments during this aging period. Like SA and Kaufmann quartz, TU Vienna quartz also loses its IN efficiency drastically over the measured time period. This shows that the decrease in IN efficiency, during aging in conditions with supersaturated Si concentration, is a common feature of all quartz samples. 
Supplement. The supplement related to this article is available online at: https://doi.org/10.5194/acp-19-6035-2019-supplement.

Author contributions. AK and CM planned the experiments. AK conducted the experiments and prepared the initial paper draft. AK, $\mathrm{CM}$, and TP contributed to the interpretation of the results and the discussion.

Competing interests. The authors declare that they have no conflict of interest.

Acknowledgements. This work was supported by the Swiss National Foundation, project number 200020_156251. We thank the following colleagues from ETH Zürich: Annette Röthlisberger and Marion Rothaupt for help in carrying out BET and XRD measurements and Michael Plötze for carrying out detailed XRD analysis; Fabian Mahrt for providing the SMPS and the APS for size distribution measurements; Nadine Borduas and Julie Tolu for their continuous help and support during ICP-MS measurements; Eszter Barthazy for carrying out SEM analysis. Jonas Fahrni and Dominik Brühwiler from Institute of Chemistry and Biotechnology (Zürich University of Applied Sciences, Wädenswil, ZHAW) for the synthesis and characterization of Stöber particles and functionalization of Alfa Aesar particles. We would also like to thank Alexei Kiselev from the Institute of Meteorology and Climate Research (Karlsruhe Institute of Technology) for providing valuable feedback on this paper.

Review statement. This paper was edited by Ryan Sullivan and reviewed by two anonymous referees.

\section{References}

Abdelmonem, A., Backus, E. H. G., Hoffmann, N., Sánchez, M. A., Cyran, J. D., Kiselev, A., and Bonn, M.: Surface-charge-induced orientation of interfacial water suppresses heterogeneous ice nucleation on $\alpha$-alumina (0001), Atmos. Chem. Phys., 17, 78277837, https://doi.org/10.5194/acp-17-7827-2017, 2017.

Alastuey, A., Querol, X., Castillo, S., Escudero, M., Avila, A., Cuevas, E., Torres, C., Romero, P.-M., Exposito, F., García, O., Pedro Diaz, J., Dingenen, R. V., and Putaud, J. P.: Characterisation of TSP and $\mathrm{PM}_{2.5}$ at Izaña and sta, Cruz de Tenerife (Canary Islands, Spain) during a Saharan dust episode (July 2002), Atmos. Environ., 39, 4715-4728, https://doi.org/10.1016/j.atmosenv.2005.04.018, 2005.

Alpert, P. A., Aller, J. Y., and Knopf, D. A.: Ice nucleation from aqueous $\mathrm{NaCl}$ droplets with and without marine diatoms, Atmos. Chem. Phys., 11, 5539-5555, https://doi.org/10.5194/acp11-5539-2011, 2011a.

Alpert, P. A., Aller, J. Y., and Knopf, D. A.: Initiation of the ice phase by marine biogenic surfaces in supersaturated gas and supercooled aqueous phases, Phys. Chem. Chem. Phys., 13, 19882-19894, https://doi.org/10.1039/C1CP21844A, 2011b.
Archuleta, C. M., DeMott, P. J., and Kreidenweis, S. M.: Ice nucleation by surrogates for atmospheric mineral dust and mineral dust/sulfate particles at cirrus temperatures, Atmos. Chem. Phys., 5, 2617-2634, https://doi.org/10.5194/acp-5-2617-2005, 2005.

Atkinson, J. D., Murray, B. J., Woodhouse, M. T., Whale, T. F., Baustian, K. J., Carslaw, K. S., Dobbie, S., O'Sullivan, D., and Malkin, T. L.: The importance of feldspar for ice nucleation by mineral dust in mixed-phase clouds, Nature, 498, 355-358, https://doi.org/10.1038/nature12278, 2013.

Augustin-Bauditz, S., Wex, H., Kanter, S., Ebert, M., Niedermeier, D., Stolz, F., Prager, A., and Stratmann, F.: The immersion mode ice nucleation behavior of mineral dusts: A comparison of different pure and surface modified dusts, Geophys. Res. Lett., 41, 7375-7382, https://doi.org/10.1002/2014GL061317, 2014.

Avila, A., Queralt-Mitjans, I., and Alarcón, M.: Mineralogical composition of African dust delivered by red rains over northeastern Spain, J. Geophys. Res.-Atmos., 102, 21977-21996, https://doi.org/10.1029/97JD00485, 1997.

Baker, M. B.: Cloud microphysics and climate, Science, 276, 10721078, https://doi.org/10.1126/science.276.5315.1072, 1997.

Baughman, R. J.: Quartz crystal growth, J. Cryst. Growth, 112, 753757, https://doi.org/10.1016/0022-0248(91)90132-O, 1991.

Belton, D. J., Deschaume, O., and Perry, C. C.: An overview of the fundamentals of the chemistry of silica with relevance to biosilicification and technological advances, FEBS J., 279, 1710-1720, https://doi.org/10.1111/j.1742-4658.2012.08531.x, 2012.

Bennett, P. C., Melcer, M. E., Siegel, D. I., and Hassett, J. P.: The dissolution of quartz in dilute aqueous solutions of organic acids at $25^{\circ} \mathrm{C}$, Geochim. Cosmochim. Ac., 52, 1521-1530, https://doi.org/10.1016/0016-7037(88)90222-0, 1988.

Berger, G., Cadore, E., Schott, J., and Dove, P. M.: Dissolution rate of quartz in lead and sodium electrolyte solutions between 25 and $300^{\circ} \mathrm{C}$ : Effect of the nature of surface complexes and reaction affinity, Geochim. Cosmochim. Ac., 58, 541-551, https://doi.org/10.1016/0016-7037(94)90487-1, 1994.

Bickmore, B. R., Wheeler, J. C., Bates, B., Nagy, K. L., and Eggett, D. L.: Reaction pathways for quartz dissolution determined by statistical and graphical analysis of macroscopic experimental data, Geochim. Cosmochim. Ac., 72, 4521-4536, https://doi.org/10.1016/j.gca.2008.07.002, 2008.

Blomfield, G. A. and Little, L. H.: Chemisorption of ammonia on silica, Can. J. Chem., 51, 1771-1781, https://doi.org/10.1139/v73-265, 1973.

Bolis, V., Fubini, B., Coluccia, S., and Mostacci, E.: Surface hydration of crystalline and amorphous silicas, J. Therm. Anal. Calorim., 30, 1283-1292, https://doi.org/10.1007/bf01914297, 1985.

Bolis, V., Fubini, B., Marchese, L., Martra, G., and Costa, D.: Hydrophilic and hydrophobic sites on dehydrated crystalline and amorphous silicas, J. Chem. Soc., 87, 497-505, https://doi.org/10.1039/FT9918700497, 1991.

Boose, Y., Sierau, B., García, M. I., Rodríguez, S., Alastuey, A., Linke, C., Schnaiter, M., Kupiszewski, P., Kanji, Z. A., and Lohmann, U.: Ice nucleating particles in the Saharan air layer, Atmos. Chem. Phys., 16, 9067-9087, https://doi.org/10.5194/acp-16-9067-2016, 2016a.

Boose, Y., Welti, A., Atkinson, J., Ramelli, F., Danielczok, A., Bingemer, H. G., Plötze, M., Sierau, B., Kanji, Z. A., and Lohmann, U.: Heterogeneous ice nucleation on dust parti- 
cles sourced from nine deserts worldwide - Part 1: Immersion freezing, Atmos. Chem. Phys., 16, 15075-15095, https://doi.org/10.5194/acp-16-15075-2016, 2016b.

Bowen, N. L.: The reaction principle in petrogenesis, J. Geol., 30, 177-198, 1922.

Bowen, N. L.: The evolution of igneous rocks, Princeton University Press, Princeton, 1928.

Brady, P. V. and Walther, J. V.: Controls on silicate dissolution rates in neutral and basic $\mathrm{pH}$ solutions at $25^{\circ} \mathrm{C}$, Geochim. Cosmochim. Ac., 53, 2823-2830, https://doi.org/10.1016/00167037(89)90160-9, 1989.

Brady, P. V. and Walther, J. V.: Kinetics of quartz dissolution at low temperatures, Chem. Geol., 82, 253-264, https://doi.org/10.1016/0009-2541(90)90084-K, 1990.

Brinker, C. J., Tallant, D. R., Roth, E. P., and Ashley, C. S.: Sol-gel transition in simple silicates: III. Structural studies during densification, J. Non-Cryst. Solids, 82, 117-126, https://doi.org/10.1016/0022-3093(86)90119-5, 1986.

Brinker, C. J., Kirkpatrick, R. J., Tallant, D. R., Bunker, B. C., and Montez, B.: NMR confirmation of strained "defects" in amorphous silica, J. Non-Cryst. Sol., 99, 418-428, https://doi.org/10.1016/0022-3093(88)90448-6, 1988.

Bunker, B. C.: Molecular mechanisms for corrosion of silica and silicate glasses, J. Non-Cryst. Solids, 179, 300-308, https://doi.org/10.1016/0022-3093(94)90708-0, 1994.

Burkert-Kohn, M., Wex, H., Welti, A., Hartmann, S., Grawe, S., Hellner, L., Herenz, P., Atkinson, J. D., Stratmann, F., and Kanji, Z. A.: Leipzig ice nucleation chamber comparison (LINC): Intercomparison of four online ice nucleation counters, Atmos. Chem. Phys., 17, 11683-11705, https://doi.org/10.5194/acp-17-116832017, 2017.

Cant, N. W. and Little, L. H.: The infrared spectrum of ammonia adsorbed on cabosil silica powder, Can. J. Chem., 43, 1252-1254, https://doi.org/10.1139/v65-170, 1965.

Cantrell, W. and Robinson, C.: Heterogeneous freezing of ammonium sulfate and sodium chloride solutions by long chain alcohols, Geophys. Res. Lett., 33, L07802, https://doi.org/10.1029/2005GL024945, 2006.

Caquineau, S., Gaudichet, A., Gomes, L., Magonthier, M.-C., and Chatenet, B.: Saharan dust: Clay ratio as a relevant tracer to assess the origin of soil-derived aerosols, Geophys. Res. Lett., 25, 983-986, https://doi.org/10.1029/98GL00569, 1998.

Caquineau, S., Gaudichet, A., Gomes, L., and Legrand, M.: Mineralogy of Saharan dust transported over northwestern tropical Atlantic ocean in relation to source regions, J. Geophys. Res., 107, 4251, https://doi.org/10.1029/2000JD000247, 2002.

Criscenti, L. J., Kubicki, J. D., and Brantley, S. L.: Silicate glass and mineral dissolution:? Calculated reaction paths and activation energies for hydrolysis of a $\mathrm{Q}^{3} \mathrm{Si}$ by $\mathrm{H}_{3} \mathrm{O}^{+}$using ab initio methods, J. Phys. Chem. A, 110, 198-206, https://doi.org/10.1021/jp044360a, 2006.

Crundwell, F. K.: On the mechanism of the dissolution of quartz and silica in aqueous solutions, ACS Omega, 2, 1116-1127, https://doi.org/10.1021/acsomega.7b00019, 2017.

DeMott, P. J., Cziczo, D. J., Prenni, A. J., Murphy, D. M., Kreidenweis, S. M., Thomson, D. S., Borys, R., and Rogers, D. C.: Measurements of the concentration and composition of nuclei for cirrus formation, P. Natl. Acad. Sci. USA, 100, 14655, https://doi.org/10.1073/pnas.2532677100, 2003 b.
DeMott, P. J., Prenni, A. J., Liu, X., Kreidenweis, S. M., Petters, M. D., Twohy, C. H., Richardson, M. S., Eidhammer, T., and Rogers, D. C.: Predicting global atmospheric ice nuclei distributions and their impacts on climate, P. Natl. Acad. Sci. USA, 107, 1121711222, https://doi.org/10.1073/pnas.0910818107, 2010.

Döbelin, N. and Kleeberg, R.: Profex: A graphical user interface for the rietveld refinement program BGMN, J. Appl. Crystallogr., 48, 1573-1580, https://doi.org/10.1107/S1600576715014685, 2015.

Donaldson, K. E. N., and Borm, P. J. A.: The quartz hazard: A variable entity, Ann. Occup. Hyg., 42, 287-294, https://doi.org/10.1093/annhyg/42.5.287, 1998.

Du, Q., Freysz, E., and Shen, Y. R.: Vibrational spectra of water molecules at quartz/water interfaces, Phys. Rev. Lett., 72, 238241, 1994.

Eischens, R. P. and Pliskin, W. A.: The infrared spectra of adsorbed molecules, in: Advances in catalysis, edited by: Eley, D. D., Frankenburg, W. G., Komarewsky, V. I., and Weisz, P. B., Academic Press, 1-56, 1958.

Field, P. R., Möhler, O., Connolly, P., Krämer, M., Cotton, R., Heymsfield, A. J., Saathoff, H., and Schnaiter, M.: Some ice nucleation characteristics of Asian and Saharan desert dust, Atmos. Chem. Phys., 6, 2991-3006, https://doi.org/10.5194/acp-6-29912006, 2006.

Folman, M.: Infra-red studies of $\mathrm{NH}_{3}$ adsorption on chlorinated porous vycor glass, Trans. Faraday Soc., 57, 2000-2006, https://doi.org/10.1039/TF9615702000, 1961.

Fubini, B.: Surface chemistery and quartz hazard, Ann. Occup. Hyg., 42, 521-530, https://doi.org/10.1093/annhyg/42.8.521, 1998.

Fubini, B., Bolis, V., and Giamello, E.: The surface chemistry of crushed quartz dust in relation to its pathogenicity, Inorg. Chim. Acta, 138, 193-197, https://doi.org/10.1016/S00201693(00)81222-0, 1987.

Fubini, B., Giamello, E., Pugliese, L., and Volante, M.: Mechanically induced defects in quartz and their impact on pathogenicity, Solid State Ionics, 32/33, 334-343, https://doi.org/10.1016/0167-2738(89)90238-5, 1989.

Fubini, B., Bolis, V., Cavenago, A., and Ugliengo, P.: Ammonia and water as probes for the surface reactivity of covalent solids: Cristobalite and silicon carbide, J. Chem. Soc., 88, 277-289, https://doi.org/10.1039/FT9928800277, 1992.

Götze, J. and Möckel, R.: Quartz: Deposits, mineralogy and analytics, Springer, Berlin, Heidelberg, 2014.

Harrison, A. D., Whale, T. F., Carpenter, M. A., Holden, M. A., Neve, L., O'Sullivan, D., Vergara Temprado, J., and Murray, B. J.: Not all feldspars are equal: A survey of ice nucleating properties across the feldspar group of minerals, Atmos. Chem. Phys., 16, 10927-10940, https://doi.org/10.5194/acp-16-109272016, 2016.

Henderson, J. H., Syers, J. K., and Jackson, M. L.: Quartz dissolution as influenced by $\mathrm{pH}$ and the presence of a disturbed surface layer, Isr. J. Chem., 8, 357-372, https://doi.org/10.1002/ijch.197000042, 1970.

House, W. A. and Orr, D. R.: Investigation of the $\mathrm{pH}$ dependence of the kinetics of quartz dissolution at 25 degree C, J. Chem. Soc., 88, 233-241, https://doi.org/10.1039/FT9928800233, 1992.

Huneeus, N., Schulz, M., Balkanski, Y., Griesfeller, J., Prospero, J., Kinne, S., Bauer, S., Boucher, O., Chin, M., Dentener, F., 
Diehl, T., Easter, R., Fillmore, D., Ghan, S., Ginoux, P., Grini, A., Horowitz, L., Koch, D., Krol, M. C., Landing, W., Liu, X., Mahowald, N., Miller, R., Morcrette, J. J., Myhre, G., Penner, J., Perlwitz, J., Stier, P., Takemura, T., and Zender, C. S.: Global dust model intercomparison in aerocom phase I, Atmos. Chem. Phys., 11, 7781-7816, https://doi.org/10.5194/acp11-7781-2011, 2011.

Isono, K. and Ikebe, Y.: On the ice-nucleating ability of rockforming minerals and soil particles, J. Meteorol. Soc. Japn. Ser. II, 38, 213-230, https://doi.org/10.2151/jmsj1923.38.5_213, 1960.

Johari, G. P., Fleissner, G., Hallbrucker, A., and Mayer, E.: Thermodynamic continuity between glassy and normal water, J. Phys. Chem., 98, 4719-4725, https://doi.org/10.1021/j100068a038, 1994.

Kandler, K., Schutz, L., Deutscher, C., Ebert, M., Hofmann, H., Jackel, S., Jaenicke, R., Knippertz, P., Lieke, K., and Massling, A.: Size distribution, mass concentration, chemical and mineralogical composition and derived optical parameters of the boundary layer aerosol at Tinfou, Morocco, during SAMUM 2006, Tellus B, 61, 32-50, 2009.

Kanji, Z. A., Sullivan, R. C., Niemand, M., DeMott, P. J., Prenni, A. J., Chou, C., Saathoff, H., and Möhler, O.: Heterogeneous ice nucleation properties of natural desert dust particles coated with a surrogate of secondary organic aerosol, Atmos. Chem. Phys., 19, 5091-5110, https://doi.org/10.5194/acp-19-5091-2019, 2019.

Kaufmann, L., Marcolli, C., Hofer, J., Pinti, V., Hoyle, C. R., and Peter, T.: Ice nucleation efficiency of natural dust samples in the immersion mode, Atmos. Chem. Phys., 16, 11177-11206, https://doi.org/10.5194/acp-16-11177-2016, 2016.

Kaufmann, L., Marcolli, C., Luo, B., and Peter, T.: Refreeze experiments with water droplets containing different types of ice nuclei interpreted by classical nucleation theory, Atmos. Chem. Phys., 17, 3525-3552, https://doi.org/10.5194/acp-173525-2017, 2017.

Kline, W. E. and Fogler, H. S.: Dissolution kinetics: Catalysis by strong acids, J. Colloid Interface Sci., 82, 93-102, https://doi.org/10.1016/0021-9797(81)90127-2, 1981.

Knauss, K. G., and Wolery, T. J.: The dissolution kinetics of quartz as a function of $\mathrm{pH}$ and time at $70^{\circ} \mathrm{C}$, Geochim. Cosmochim. Ac., 52, 43-53, https://doi.org/10.1016/0016-7037(88)90055-5, 1988.

Knopf, D. A. and Forrester, S. M.: Freezing of water and aqueous $\mathrm{NaCl}$ droplets coated by organic monolayers as a function of surfactant properties and water activity, J. Phys. Chem. A, 115, 5579-5591, https://doi.org/10.1021/jp2014644, 2011.

Knopf, D. A. and Alpert, P. A.: A water activity based model of heterogeneous ice nucleation kinetics for freezing of water and aqueous solution droplets, Farad. Discussions, 165, 513-534, https://doi.org/10.1039/C3FD00035D, 2013.

Kolb, C. E., Cox, R. A., Abbatt, J. P. D., Ammann, M., Davis, E. J., Donaldson, D. J., Garrett, B. C., George, C., Griffiths, P. T., Hanson, D. R., Kulmala, M., McFiggans, G., Pöschl, U., Riipinen, I., Rossi, M. J., Rudich, Y., Wagner, P. E., Winkler, P. M., Worsnop, D. R., and O' Dowd, C. D.: An overview of current issues in the uptake of atmospheric trace gases by aerosols and clouds, Atmos. Chem. Phys., 10, 10561-10605, https://doi.org/10.5194/acp-1010561-2010, 2010.
Koop, T., Luo, B., Tsias, A., and Peter, T.: Water activity as the determinant for homogeneous ice nucleation in aqueous solutions, Nature, 406, 611-614, 2000.

Kulkarni, G., Fan, J., Comstock, J. M., Liu, X., and Ovchinnikov, M.: Laboratory measurements and model sensitivity studies of dust deposition ice nucleation, Atmos. Chem. Phys., 12, 72957308, https://doi.org/10.5194/acp-12-7295-2012, 2012.

Kumar, A., Marcolli, C., Luo, B., and Peter, T.: Ice nucleation activity of silicates and aluminosilicates in pure water and aqueous solutions - Part 1: The K-feldspar microcline, Atmos. Chem. Phys., 18, 7057-7079, https://doi.org/10.5194/acp18-7057-2018, 2018a.

Kumar, A., Marcolli, C., and Peter, T.: Research Data supporting "Ice nucleation activity of silicates and aluminosilicates in pure water and aqueous solutions, Part 2 - Quartz and amorphous silica", https://doi.org/10.3929/ethz-b-000286931, last access: 1 November 2018 b.

Kumar, A., Marcolli, C., and Peter, T.: Ice nucleation activity of silicates and aluminosilicates in pure water and aqueous solutions - Part 3: Aluminosilicates, Atmos. Chem. Phys., 19, 6059-6084, https://doi.org/10.5194/acp-19-6059-2019, 2019.

Laurent, B., Marticorena, B., Bergametti, G., and Mei, F.: Modeling mineral dust emissions from chinese and mongolian deserts, Glob. Planet. Change, 52, 121-141, https://doi.org/10.1016/j.gloplacha.2006.02.012, 2006.

Laurent, B., Marticorena, B., Bergametti, G., Léon, J. F., and Mahowald, N. M.: Modeling mineral dust emissions from the sahara desert using new surface properties and soil database, J. Geophys. Res.-Atmos., 113, D14218, https://doi.org/10.1029/2007JD009484, 2008.

Li, C.-Z. and Nelson, P. F.: Interactions of quartz, zircon sand and stainless steel with ammonia: Implications for the measurement of ammonia at high temperatures, Fuel, 75, 525-526, https://doi.org/10.1016/0016-2361(95)00256-1, 1996.

Makoto, H. and Motoji, I.: Effects of UV exposure on e' centers formed in crushed quartz grains, Jpn. J. Appl. Phys., 35, 44634467, https://doi.org/10.1143/JJAP.35.4463, 1996.

Mapes, J. E. and Eischens, R. P.: The infrared spectra of ammonia chemisorbed on cracking catalysts, J. Phys. Chem., 58, 10591062, https://doi.org/10.1021/j150522a002, 1954.

Marcolli, C., Gedamke, S., Peter, T., and Zobrist, B.: Efficiency of immersion mode ice nucleation on surrogates of mineral dust, Atmos. Chem. Phys., 7, 5081-5091, https://doi.org/10.5194/acp7-5081-2007, 2007.

Marcolli, C., Nagare, B., Welti, A., and Lohmann, U.: Ice nucleation efficiency of AgI: Review and new insights, Atmos. Chem. Phys., 16, 8915-8937, https://doi.org/10.5194/acp-168915-2016, 2016.

Matsuki, A., Iwasaka, Y., Shi, G. Y., Zhang, D. Z., Trochkine, D., Yamada, M., Kim, Y. S., Chen, B., Nagatani, T., and Miyazawa, T.: Morphological and chemical modification of mineral dust: Observational insight into the heterogeneous uptake of acidic gases, Geophys. Res. Lett., 32, L22806, https://doi.org/10.1029/2005GL024176, 2005.

Morrow, B. A. and Cody, I. A.: Infrared studies of reactions on oxide surfaces, 5. Lewis acid sites on dehydroxylated silica, J. Phys. Chem., 80, 1995-1998, https://doi.org/10.1021/j100559a009, 1976. 
Morrow, B. A., Cody, I. A., and Lee, L. S. M.: Infrared studies of reactions on oxide surfaces, 7. Mechanism of the adsorption of water and ammonia on dehydroxylated silica, J. Phys. Chem., 80, 2761-2767, https://doi.org/10.1021/j100566a009, 1976.

Murray, B. J., Broadley, S. L., Wilson, T. W., Atkinson, J. D., and Wills, R. H.: Heterogeneous freezing of water droplets containing kaolinite particles, Atmos. Chem. Phys., 11, 4191-4207, https://doi.org/10.5194/acp-11-4191-2011, 2011.

Murray, B. J., O'Sullivan, D., Atkinson, J. D., and Webb, M. E.: Ice nucleation by particles immersed in supercooled cloud droplets, Chem. Soc. Rev., 41, 6519-6554, https://doi.org/10.1039/C2CS35200A, 2012.

Musso, F., Sodupe, M., Corno, M., and Ugliengo, P.: H-Bond features of fully hydroxylated surfaces of crystalline silica polymorphs: A periodic B3LYP study, J. Phys. Chem. C, 113, 1787617884, https://doi.org/10.1021/jp905325m, 2009.

Musso, F., Ugliengo, P., and Sodupe, M.: Do H-Bond features of silica surfaces affect the $\mathrm{H}_{2} \mathrm{O}$ and $\mathrm{NH}_{3}$ adsorption? Insights from periodic B3LYP calculations, J. Phys. Chem. A, 115, 1122111228, https://doi.org/10.1021/jp203988j, 2011.

Musso, F., Mignon, P., Ugliengo, P., and Sodupe, M.: Cooperative effects at water-crystalline silica interfaces strengthen surface silanol hydrogen bonding. An ab initio molecular dynamics study, Phys. Chem. Chem. Phys., 14, 10507-10514, https://doi.org/10.1039/C2CP40756F, 2012.

Muster, T. H., Prestidge, C. A., and Hayes, R. A.: Water adsorption kinetics and contact angles of silica particles, Colloids Surf. Physicochem. Eng. Aspects, 176, 253-266, https://doi.org/10.1016/S0927-7757(00)00600-2, 2001.

Niedermeier, D., Hartmann, S., Clauss, T., Wex, H., Kiselev, A., Sullivan, R. C., DeMott, P. J., Petters, M. D., Reitz, P., Schneider, J., Mikhailov, E., Sierau, B., Stetzer, O., Reimann, B., Bundke, U., Shaw, R. A., Buchholz, A., Mentel, T. F., and Stratmann, F.: Experimental study of the role of physicochemical surface processing on the in ability of mineral dust particles, Atmos. Chem. Phys., 11, 11131-11144, https://doi.org/10.5194/acp-11-111312011, 2011.

O'Sullivan, D., Murray, B. J., Malkin, T. L., Whale, T. F., Umo, N. S., Atkinson, J. D., Price, H. C., Baustian, K. J., Browse, J., and Webb, M. E.: Ice nucleation by fertile soil dusts: relative importance of mineral and biogenic components, Atmos. Chem. Phys., 14, 1853-1867, https://doi.org/10.5194/acp-141853-2014, 2014.

Peckhaus, A., Kiselev, A., Hiron, T., Ebert, M., and Leisner, T.: A comparative study of K-rich and $\mathrm{Na} / \mathrm{Ca}$-rich feldspar icenucleating particles in a nanoliter droplet freezing assay, Atmos. Chem. Phys., 16, 11477-11496, https://doi.org/10.5194/acp-1611477-2016, 2016.

Pedevilla, P., Fitzner, M., and Michaelides, A.: What makes a good descriptor for heterogeneous ice nucleation on OH-patterned surfaces, Phys. Rev. B, 96, 115441, https://doi.org/10.1103/PhysRevB.96.115441, 2017.

Peri, J. B.: Infrared study of $\mathrm{OH}$ and $\mathrm{NH}_{2}$ groups on the surface of a dry silica aerogel, J. Phys. Chem., 70, 2937-2945, https://doi.org/10.1021/j100881a037, 1966.

Peri, J. B. and Hannan, R. B.: Surface hydroxyl groups on $\gamma$-alumina1, J. Phys. Chem., 64, 1526-1530, https://doi.org/10.1021/j100839a044, 1960.
Perry, C. C.: Chemical studies of biogenic silica, in: Biomineralisation, chemical and biological perspectives, edited by: Mann, S., Webb, J., and Williams, R. J. P., VCH, Weinheim, 233-256, 1989.

Perry, C. C.: Silicification: The processes by which organisms capture and mineralize silica, Rev. Mineral. Geochem., 54, 291-327, https://doi.org/10.2113/0540291, 2003.

Pinti, V., Marcolli, C., Zobrist, B., Hoyle, C. R., and Peter, T.: Ice nucleation efficiency of clay minerals in the immersion mode, Atmos. Chem. Phys., 12, 5859-5878, https://doi.org/10.5194/acp-12-5859-2012, 2012.

Poulsen, H. F., Neuefeind, J., Neumann, H. B., Schneider, J. R., and Zeidler, M. D.: Amorphous silica studied by high energy X-ray diffraction, J. Non-Cryst. Solids, 188, 63-74, https://doi.org/10.1016/0022-3093(95)00095-X, 1995.

Prospero, J. M.: Long-range transport of mineral dust in the global atmosphere: Impact of African dust on the environment of the Southeastern United States, P. Natl. Acad. Sci. USA, 96, 3396, https://doi.org/10.1073/pnas.96.7.3396, 1999.

Pruppacher, H. R. and Sänger, R.: Mechanismus der vereisung unterkühlter wassertropfen durch disperse keimsubstanzen, Z. Angew. Math. Phys., 6, 407-416, https://doi.org/10.1007/bf01589767, 1955.

Pruppacher, H. R. and Klett, J. D.: Microphysics of clouds and precipitation, Kluwer Academic Publishers, Dordrecht, the Netherlands, 1994.

Reitz, P., Spindler, C., Mentel, T. F., Poulain, L., Wex, H., Mildenberger, K., Niedermeier, D., Hartmann, S., Clauss, T., Stratmann, F., Sullivan, R. C., DeMott, P. J., Petters, M. D., Sierau, B., and Schneider, J.: Surface modification of mineral dust particles by sulphuric acid processing: Implications for ice nucleation abilities, Atmos. Chem. Phys., 11, 7839-7858, https://doi.org/10.5194/acp-11-7839-2011, 2011.

Richmond, G. L.: Molecular bonding and interactions at aqueous surfaces as probed by vibrational sum frequency spectroscopy, Chem. Rev., 102, 2693-2724, https://doi.org/10.1021/cr0006876, 2002.

Rigg, Y. J., Alpert, P. A., and Knopf, D. A.: Immersion freezing of water and aqueous ammonium sulfate droplets initiated by humic-like substances as a function of water activity, Atmos. Chem. Phys., 13, 6603-6622, https://doi.org/10.5194/acp13-6603-2013, 2013.

Salam, A., Lohmann, U., and Lesins, G.: Ice nucleation of ammonia gas exposed montmorillonite mineral dust particles, Atmos. Chem. Phys., 7, 3923-3931, https://doi.org/10.5194/acp-7-39232007, 2007.

Salam, A., Lesins, G., and Lohmann, U.: Laboratory study of heterogeneous ice nucleation in deposition mode of montmorillonite mineral dust particles aged with ammonia, sulfur dioxide, and ozone at polluted atmospheric concentrations, Air Qual. Atmos. Health, 1, 135-142, https://doi.org/10.1007/s11869-008-0019-6, 2008.

Schepanski, K., Tegen, I., and Macke, A.: Saharan dust transport and deposition towards the tropical northern Atlantic, Atmos. Chem. Phys., 9, 1173-1189, https://doi.org/10.5194/acp-9-11732009, 2009.

Schwartzentruber, J., Fürst, W., and Renon, H.: Dissolution of quartz into dilute alkaline solutions at $90^{\circ} \mathrm{C}$ : A ki- 
netic study, Geochim. Cosmochim. Ac., 51, 1867-1874, https://doi.org/10.1016/0016-7037(87)90177-3, 1987.

Speedy, R. J.: Thermodynamic properties of supercooled water at $1 \mathrm{~atm}$, J. Phys. Chem., 91, 3354-3358, https://doi.org/10.1021/j100296a049, 1987.

Storelvmo, T., Hoose, C., and Eriksson, P.: Global modeling of mixed-phase clouds: The albedo and lifetime effects of aerosols, J. Geophys. Res.-Atmos., 116, D05207, https://doi.org/10.1029/2010JD014724, 2011.

Sullivan, R. C., Miñambres, L., DeMott, P. J., Prenni, A. J., Carrico, C. M., Levin, E. J. T., and Kreidenweis, S. M.: Chemical processing does not always impair heterogeneous ice nucleation of mineral dust particles, Geophys. Res. Lett., 37, L24805, https://doi.org/10.1029/2010GL045540, 2010a.

Sullivan, R. C., Petters, M. D., DeMott, P. J., Kreidenweis, S. M., Wex, H., Niedermeier, D., Hartmann, S., Clauss, T., Stratmann, F., Reitz, P., Schneider, J., and Sierau, B.: Irreversible loss of ice nucleation active sites in mineral dust particles caused by sulphuric acid condensation, Atmos. Chem. Phys., 10, 1147111487, https://doi.org/10.5194/acp-10-11471-2010, 2010b.

Tamahrajah, J. and Brehm, A.: Preliminary kinetic data of silicic acid species prior to the formation of exoskeletal structures, Mar. Chem., 181, 18-24, https://doi.org/10.1016/j.marchem.2016.03.001, 2016.

Tobo, Y., DeMott, P. J., Hill, T. C. J., Prenni, A. J., SwobodaColberg, N. G., Franc, G. D., and Kreidenweis, S. M.: Organic matter matters for ice nuclei of agricultural soil origin, Atmos. Chem. Phys., 14, 8521-8531, https://doi.org/10.5194/acp14-8521-2014, 2014.

Tribello, G. A., Slater, B., Zwijnenburg, M. A., and Bell, R. G.: Isomorphism between ice and silica, Phys. Chem. Chem. Phys., 12, 8597-8606, https://doi.org/10.1039/B916367K, 2010.

Tsyganenko, A. A., Pozdnyakov, D. V., and Filimonov, V. N.: Infrared study of surface species arising from ammonia adsorption on oxide surfaces, J. Mol. Struct., 29, 299-318, https://doi.org/10.1016/0022-2860(75)85038-1, 1975.

Turci, F., Pavan, C., Leinardi, R., Tomatis, M., Pastero, L., Garry, D., Anguissola, S., Lison, D., and Fubini, B.: Revisiting the paradigm of silica pathogenicity with synthetic quartz crystals: The role of crystallinity and surface disorder, Part. Fibre Toxicol., 13, https://doi.org/10.1186/s12989-016-0136-6, 2016.

Uno, I., Eguchi, K., Yumimoto, K., Takemura, T., Shimizu, A., Uematsu, M., Liu, Z., Wang, Z., Hara, Y., and Sugimoto, N.: Asian dust transported one full circuit around the globe, Nat. Geosci., 2, 557-560, https://doi.org/10.1038/ngeo583, 2009.

Usher, C. R., Michel, A. E., and Grassian, V. H.: Reactions on mineral dust, Chem. Rev., 103, 4883-4940, https://doi.org/10.1021/cr020657y, 2003.

Vali, G.: Interpretation of freezing nucleation experiments: Singular and stochastic; sites and surfaces, Atmos. Chem. Phys., 14, 5271-5294, https://doi.org/10.5194/acp-14-5271-2014, 2014.

Vali, G., DeMott, P. J., Möhler, O., and Whale, T. F.: Technical note: A proposal for ice nucleation terminology, Atmos. Chem. Phys., 15, 10263-10270, https://doi.org/10.5194/acp-15-102632015, 2015.

Vidyadhar, A. and Hanumantha Rao, K.: Adsorption mechanism of mixed cationic/anionic collectors in feldspar-quartz flotation system, J. Colloid Interface Sci., 306, 195-204, https://doi.org/10.1016/j.jcis.2006.10.047, 2007.
Walther, J. V. and Helgeson, H. C.: Calculation of the thermodynamic properties of aqueous silica and the solubility of quartz and its polymorphs at high pressures and temperatures, Am. J. Sci., 277, 1315-1351, https://doi.org/10.2475/ajs.277.10.1315, 1977.

Wei, X., Miranda, P. B., Zhang, C., and Shen, Y. R.: Sum-frequency spectroscopic studies of ice interfaces, Phys. Rev. B, 66, 085401, https://doi.org/10.1103/PhysRevB.66.085401, 2002.

Wex, H., DeMott, P. J., Tobo, Y., Hartmann, S., Rösch, M., Clauss, T., Tomsche, L., Niedermeier, D., and Stratmann, F.: Kaolinite particles as ice nuclei: learning from the use of different kaolinite samples and different coatings, Atmos. Chem. Phys., 14, 55295546, https://doi.org/10.5194/acp-14-5529-2014, 2014.

Whale, T. F., Holden, M. A., Kulak, A. N., Kim, Y.-Y., Meldrum, F. C., Christenson, H. K., and Murray, B. J.: The role of phase separation and related topography in the exceptional ice-nucleating ability of alkali feldspars, Phys. Chem. Chem. Phys., 19, 3118631193, https://doi.org/10.1039/C7CP04898J, 2017.

Whale, T. F., Holden, M. A., Wilson, T. W., O'Sullivan, D., and Murray, B. J.: The enhancement and suppression of immersion mode heterogeneous ice-nucleation by solutes, Chem. Sci., 9, 4142-4151, https://doi.org/10.1039/C7SC05421A, 2018.

Wright, L. B. and Walsh, T. R.: First-principles molecular dynamics simulations of $\mathrm{NH}_{4}^{+}$and $\mathrm{CH}_{3} \mathrm{COO}^{-}$adsorption at the aqueous quartz interface, J. Chem. Phys., 137, 224702, https://doi.org/10.1063/1.4769727, 2012.

Xiao, Y. and Lasaga, A. C.: Ab initio quantum mechanical studies of the kinetics and mechanisms of silicate dissolution: $\mathrm{H}^{+}\left(\mathrm{H}_{3} \mathrm{O}^{+}\right)$catalysis, Geochim. Cosmochim. Ac., 58, 53795400, https://doi.org/10.1016/0016-7037(94)90237-2, 1994.

Zhdanov, S. P., Kosheleva, L. S., and Titova, T. I.: IR study of hydroxylated silica, Langmuir, 3, 960-967, https://doi.org/10.1021/la00078a014, 1987.

Zhuravlev, L. T.: The surface chemistry of amorphous silica. Zhuravlev model, Colloids Surf. Physicochem. Eng. Aspects, 173, 1-38, https://doi.org/10.1016/S0927-7757(00)00556-2, 2000.

Zimmermann, F., Weinbruch, S., Schutz, L., Hofmann, H., Ebert, M., Kandler, K., and Worringen, A.: Ice nucleation properties of the most abundant mineral dust phases, J. Geophys. Res., 113, D23204, https://doi.org/10.1029/2008JD010655, 2008.

Zobrist, B., Marcolli, C., Koop, T., Luo, B. P., Murphy, D. M., Lohmann, U., Zardini, A. A., Krieger, U. K., Corti, T., Cziczo, D. J., Fueglistaler, S., Hudson, P. K., Thomson, D. S., and Peter, T.: Oxalic acid as a heterogeneous ice nucleus in the upper troposphere and its indirect aerosol effect, Atmos. Chem. Phys., 6, 3115-3129, https://doi.org/10.5194/acp-6-3115-2006, 2006.

Zobrist, B., Marcolli, C., Peter, T., and Koop, T.: Heterogeneous ice nucleation in aqueous solutions:? The role of water activity, J. Phys. Chem. A, 112, 3965-3975, https://doi.org/10.1021/jp7112208, 2008.

Zolles, T., Burkart, J., Häusler, T., Pummer, B., Hitzenberger, R., and Grothe, H.: Identification of ice nucleation active sites on feldspar dust particles, J. Phys. Chem. A, 119, 2692-2700, https://doi.org/10.1021/jp509839x, 2015.

Zuberi, B., Bertram, A. K., Cassa, C. A., Molina, L. T., and Molina, M. J.: Heterogeneous nucleation of ice in $\left(\mathrm{NH}_{4}\right)_{2} \mathrm{SO}_{4}-\mathrm{H}_{2} \mathrm{O}$ particles with mineral dust immersions, Geophys. Res. Lett., 29, https://doi.org/10.1029/2001GL014289, 2002. 
Zuend, A., Marcolli, C., Luo, B. P., and Peter, T.: A thermodynamic model of mixed organic-inorganic aerosols to predict activity coefficients, Atmos. Chem. Phys., 8, 4559-4593, https://doi.org/10.5194/acp-8-4559-2008, 2008.

Zuend, A., Marcolli, C., Booth, A. M., Lienhard, D. M., Soonsin, V., Krieger, U. K., Topping, D. O., McFiggans, G., Peter, T., and Seinfeld, J. H.: New and extended parameterization of the thermodynamic model AIOMFAC: Calculation of activity coefficients for organic-inorganic mixtures containing carboxyl, hydroxyl, carbonyl, ether, ester, alkenyl, alkyl, and aromatic functional groups, Atmos. Chem. Phys., 11, 9155-9206, https://doi.org/10.5194/acp-11-9155-2011, 2011. 